\title{
Optimal Detector Randomization for Multiuser Communications Systems
}

\author{
Mehmet Emin Tutay, Student Member, IEEE, Sinan Gezici, Senior Member, IEEE, \\ and Orhan Arikan, Member, IEEE
}

\begin{abstract}
Optimal detector randomization is studied for the downlink of a multiuser communications system, in which users can perform time-sharing among multiple detectors. A formulation is provided to obtain optimal signal amplitudes, detectors, and detector randomization factors. It is shown that the solution of this joint optimization problem can be calculated in two steps, resulting in significant reduction in computational complexity. It is proved that the optimal solution is achieved via randomization among at most $\min \left\{K, N_{\mathrm{d}}\right\}$ detector sets, where $K$ is the number of users and $N_{\mathrm{d}}$ is the number of detectors at each receiver. Lower and upper bounds are derived on the performance of optimal detector randomization, and it is proved that the optimal detector randomization approach can reduce the worst-case average probability of error of the optimal approach that employs a single detector for each user by up to $K$ times. Various sufficient conditions are obtained for the improvability and nonimprovability via detector randomization. In the special case of equal crosscorrelations and noise powers, a simple solution is developed for the optimal detector randomization problem, and necessary and sufficient conditions are presented for the uniqueness of that solution. Numerical examples are provided to illustrate the improvements achieved via detector randomization.
\end{abstract}

Index Terms-Detection, multiuser, randomization, probability of error, time-sharing, minimax.

\section{INTRODUCTION}

$\mathbf{R}$ ECENTLY, the effects of randomization or time-sharing have been investigated in various studies such as [1][13]. In [1], the convexity properties of error probability in terms of signal and noise power are investigated for binary-valued scalar signals over additive unimodal noise channels under an average power constraint. Based on the convexity results, the scenarios in which power randomization can or cannot be useful for improving error performance are determined, and optimal strategies for jammer power randomization are developed. The study in [2] generalizes the results of [1] by exploring the convexity properties of the error probability for constellations with arbitrary shape, order, and dimensionality for a maximum likelihood (ML) detector in the presence of additive Gaussian noise with no

Manuscript received February 1, 2013; revised May 9, 2013. The editor coordinating the review of this paper and approving it for publication was D. Gunduz.

This research was supported in part by the National Young Researchers Career Development Programme (project no. 110E245) of the Scientific and Technological Research Council of Turkey (TUBITAK).

The authors are with the Department of Electrical and Electronics Engineering, Bilkent University, Bilkent, Ankara 06800, Turkey (e-mail: \{tutay, gezici, oarikan\}@ee.bilkent.edu.tr).

Digital Object Identifier 10.1109/TCOMM.2013.053013.130099 fading and with frequency-flat slowly fading channels. For communications systems that operate over time-invariant nonGaussian channels [14], randomization (time-sharing) among multiple signal constellations can improve performance of a given receiver in terms of error probability. Specifically, it is shown in [3] that randomization among up to three distinct signal constellations can reduce the average probability of error of a communications system that operates under second and fourth moment constraints. In addition, [4] investigates the joint optimization of the signal constellation randomization and detector design under an average power constraint and shows that the use of at most two distinct signal constellations and the corresponding maximum a posteriori probability (MAP) detector minimizes the average probability of error. In [5], optimal signal constellation randomization is studied for the downlink of a multiuser communications system considering given detectors at the receivers, and an approximate solution is provided based on convex relaxation. In addition, asymptotical improvements that can be achieved via constellation randomization are quantified when symmetric signaling and sign detectors are employed. In a different context, time-varying or random signal constellations are utilized in [15]-[20] for the purpose of enhancing error performance or achieving diversity.

Another technique for enhancing error performance of some communications systems that operate over time-invariant channels is to perform detector randomization, which involves the use of multiple detectors at the receiver with certain probabilities (certain fractions of time) [6]-[8], [21], [22]. In other words, a receiver can randomize (time-share) among multiple detectors in order to reduce the average probability of error. In [6], randomization between two antipodal signal pairs and the corresponding MAP detectors is performed for an average power constrained binary communications system, and significant performance improvements are observed as a result of detector randomization in some cases in the presence of symmetric Gaussian mixture noise. In [7], the results in [6] and [4] are extended by considering both detector randomization and signal constellation randomization for an average power constrained $M$-ary communications system. It is proved that the joint optimization of detector and signal constellation randomization results in a randomization between at most two MAP detectors corresponding to two deterministic signal constellations. The study in [7] is extended to the NeymanPearson (NP) framework in [21] by considering a power constrained on-off keying communications systems. As discussed in [23], detector randomization can be regarded as a gener- 
alization of noise enhanced detection with a fixed detector [9], [13]. In addition, when variable detectors are considered, noise enhanced detection and detector randomization can be considered as alternative approaches. ${ }^{1}$ In [8], probability distributions of optimal additive noise components are investigated for variable detectors, and the optimal randomization between detector and additive noise pairs is investigated for optimal noise enhancement.

Although detector randomization has recently been investigated, e.g., in [6]-[8], [21], no previous studies have considered detector randomization for multiuser communications systems. In this paper, we study optimal detector randomization for multiuser communications systems. In particular, we consider the downlink of a direct sequence spread spectrum (DSSS) communications system under an average power constraint, and propose an optimization problem to obtain optimal signal amplitudes (corresponding to information symbols for different users), detectors, and detector randomization factors (probabilities) that minimize the worstcase (maximum) average probability of error of the users. Since this joint optimization problem is quite complex in its original formulation, a low-complexity approach is developed in order to obtain the optimal solution in two steps, where the optimal signal amplitudes and detector randomization factors are calculated in the first step, and the corresponding ML detectors are obtained in the second step. Also, it is shown that the optimal solution requires randomization among at most $\min \left\{K, N_{\mathrm{d}}\right\}$ detectors for each user, where $K$ is the number of users and $N_{\mathrm{d}}$ is the number of detectors at each receiver. In addition, the performance of the optimal detector randomization approach is investigated, and a lower bound is presented for the minimum worst-case average probability of error. It is proved that the optimal detector randomization approach can improve the performance of the optimal approach that employs a single detector for each user (i.e., no detector randomization) by up to $K$ times. Sufficient conditions are derived for the improvability and nonimprovability via detector randomization. Furthermore, in the special case of equal crosscorrelations and noise powers, a simple solution is proposed for the optimal detector randomization problem, and necessary and sufficient conditions are obtained for the uniqueness of that solution. Finally, numerical examples are presented in order to illustrate the improvements achieved via detector randomization. Although the results in this study are obtained for the downlink of a binary DSSS system, possible extensions to uplink scenarios and $M$-ary systems are discussed in Section VI.

It should be emphasized that detector randomization in this study is designed for time-invariant channels; equivalently, detector randomization is performed for each channel realization assuming that channel statistics do not change for a certain number of symbols [6], [7], [21]. Therefore, the proposed approach is different from power control (and detector adaptation) algorithms that are developed for varying channel conditions [24]-[26]. In addition, randomized power

\footnotetext{
${ }^{1}$ The main difference is that an additive noise component is employed at the detector in the noise enhanced detection approach whereas the transmitted signal values are adapted according to the detector randomization strategy in the detector randomization approach.
}

control algorithms in the literature, such as [27]-[32], employ significantly different approaches than that in this study. For example, a random power control algorithm is proposed in [29], where the transmitter selects its power level randomly from a uniform distribution. This approach is shown to improve network connectivity over the fixed power control approach for static channels. In [31], random power allocation is performed according to a certain probability distribution; namely, the transmit power is modeled by a truncated inverted exponential distribution, and the parameter of this distribution is updated at certain intervals based on feedback. In addition, [27] considers a scenario in which transmit powers are selected from a discrete set of power levels, namely, zero and peak power, and optimal power randomization strategies are developed under that specification for a two-hop interference channel.

The remainder of the paper is organized as follows. In Section II, the system model is introduced and receiver structures are described. In Section III, the optimal detector randomization problem is formulated, and a low-complexity approach is presented. Analysis of optimal detector randomization is performed in Section IV, and lower bounds and upper bounds are obtained on the performance of optimal detector randomization. In addition, various conditions for improvability or nonimprovability via detector randomization are derived, and simple solution is provided for equal crosscorrelations and noise powers. Numerical examples are presented in Section V. In Section VI, concluding remarks are made and possible extensions to uplink scenarios and $M$-ary systems are discussed.

\section{SySTEM MODEL}

Consider the downlink of a multiuser communications system in which the transmitter (e.g., base station or access point) sends information bearing signals to $K$ users simultaneously via code division multiple access (CDMA). In addition, assume that the users can perform detector randomization [6], [7] in coordination with the transmitter by employing different detectors for certain fractions of time. In particular, suppose that each user can time-share (randomize) among $N_{\mathrm{d}}$ detectors; namely, user $k$ employs detector $\phi_{1}^{(k)}$ for the first $N_{\mathrm{s}, 1}$ symbols, detector $\phi_{2}^{(k)}$ for the next $N_{\mathrm{s}, 2}$ symbols, $\ldots$, and detector $\phi_{N_{\mathrm{d}}}^{(k)}$ for the last $N_{\mathrm{s}, N_{\mathrm{d}}}$ symbols $^{2}$, where $k \in\{1,2, \ldots, K\}$. The described scenario is also depicted in Fig. 1, which illustrates a $K$-user system with $N_{\mathrm{d}}$ detectors for each user.

For the downlink of a DSSS binary ${ }^{3}$ communications system as in Fig. 1, the baseband model of the transmitted signal can be expressed as

$$
p(t)=\sum_{k=1}^{K} S_{k, l}^{\left(i_{k}\right)} c_{k}(t)
$$

\footnotetext{
${ }^{2}$ Such a coordination can be achieved in practice by employing a communications protocol that informs the users about this randomization (timesharing) structure by including the related information in the header of the communications packet [7].

${ }^{3}$ As mentioned in Section VI, the results can be extended to $M$-ary communications systems as well.
} 


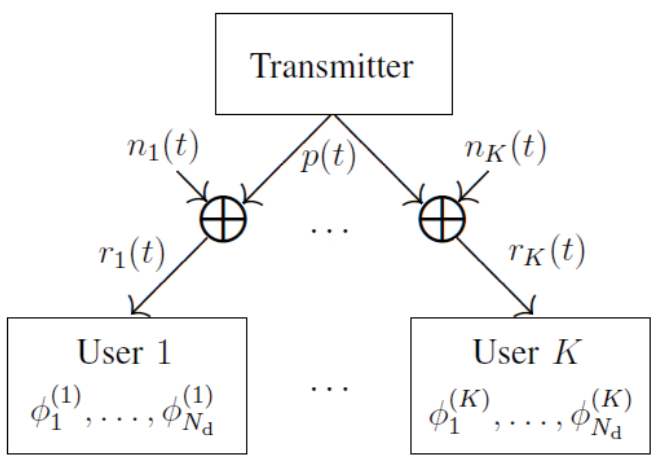

Fig. 1. System model. The transmitter sends information bearing signals to $K$ users over additive noise channels, and each user estimates the transmitted symbol by performing detector randomization among $N_{\mathrm{d}}$ detectors.

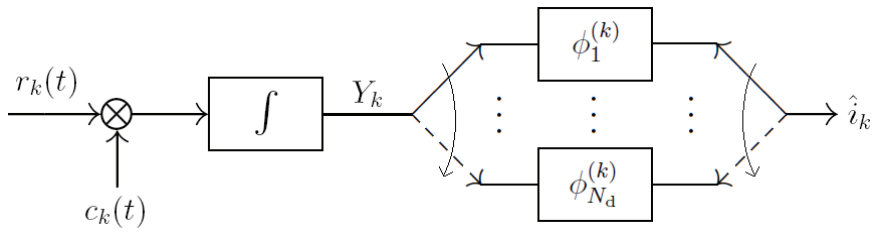

Fig. 2. Receiver structure for user $k$. The received signal is first despread by the pseudo-noise signal, and the resulting signal, $Y_{k}$, is processed by one of the detectors according to a detector randomization strategy.

for $l \in\left\{1, \ldots, N_{\mathrm{d}}\right\}$ and $i_{k} \in\{0,1\}$, where $K$ is the number of users, $S_{k, l}^{\left(i_{k}\right)}$ denotes the transmitted signal amplitude for information bit $i_{k}$ that is intended for detector $l$ of user $k$, and $c_{k}(t)$ is the real pseudo-noise signal for user $k$ [5]. Pseudo-noise signals are employed to spread the spectra of users' signals and provide multiple-access capability [33]. It is assumed that the prior probabilities of bit 0 and bit 1 are equal to 0.5 for all users, and that the information bits of different users are independent.

The signal in (1) is transmitted to $K$ users over the additive noise channels as in Fig. 1, and the received signal at user $k$ is modeled as

$$
r_{k}(t)=\sum_{j=1}^{K} S_{j, l}^{\left(i_{j}\right)} c_{j}(t)+n_{k}(t),
$$

for $k=1, \ldots, K$, where $n_{k}(t)$ is the noise at the receiver of user $k$, which is a zero-mean white Gaussian process with spectral density $\sigma_{k}^{2}$. The noise processes at different receivers are supposed to be independent. Although a simple additive noise model is employed in (2), multipath channels with slow frequency-flat fading can also be incorporated into the model under the assumption of perfect channel estimation by adjusting the average powers of the noise components in (2), equivalently, the $\sigma_{k}^{2}$ terms, accordingly [3], [5].

The receiver structure for user $k$ is illustrated in Fig. 2. The received signal $r_{k}(t)$ in (2) is first correlated with the pseudonoise signal for user $k, c_{k}(t)$. Then, the correlator output is processed by one of the detectors according to the detector randomization strategy and the transmitted bit of user $k$ is estimated. (Although $N_{\mathrm{d}}$ detectors are shown in Fig. 2, the receiver can also be implemented by adapting the parameters of one detector over time.) From (2) and Fig. 2, the correlator output for user $k, Y_{k}$, can be expressed as

$$
Y_{k}=S_{k, l}^{\left(i_{k}\right)}+\sum_{\substack{j=1 \\ j \neq k}}^{K} \rho_{k, j} S_{j, l}^{\left(i_{j}\right)}+N_{k},
$$

for $k=1, \ldots, K$, where $\rho_{k, j} \triangleq \int c_{k}(t) c_{j}(t) d t$ denotes the crosscorrelation between the pseudo-noise signals for user $k$ and $j$ (it is assumed that $\rho_{k, k}=1$ for $k=1, \ldots, K$ ), and $N_{k} \triangleq \int n_{k}(t) c_{k}(t) d t$ is the noise component. The noise components $N_{1}, \ldots, N_{K}$ form a sequence of independent zero-mean Gaussian random variables with variances, $\sigma_{1}^{2}, \ldots, \sigma_{K}^{2}$, respectively [5]. It is noted from the expression for $Y_{k}$ in (3) that the first term corresponds to the desired signal component, the second term denotes the multiple-access interference (MAI), and the last term is the noise component.

As shown in Fig. 2, the correlator output in (3) is processed by detectors $\phi_{1}^{(k)}, \ldots, \phi_{N_{\mathrm{d}}}^{(k)}$ according to a detector randomization strategy, and an estimate of the transmitted information bit, $\hat{i}_{k}$, is generated. Mathematically, for a given correlator output $Y_{k}=y_{k}$, the bit estimate is obtained as

$$
\hat{i}_{k}=\phi_{l}^{(k)}\left(y_{k}\right)= \begin{cases}1, & \text { if } y_{k} \in \Gamma_{l}^{(k)} \\ 0, & \text { otherwise }\end{cases}
$$

if the $l^{\text {th }}$ detector is employed for user $k$, where $l \in$ $\left\{1, \ldots, N_{\mathrm{d}}\right\}$ and $k \in\{1, \ldots, K\}$. In (4), $\Gamma_{l}^{(k)}$ denotes the decision region in which bit 1 is selected by the $l^{\text {th }}$ detector of user $k$. The receiver of user $k$ can perform randomization among these $N_{\mathrm{d}}$ detectors in order to optimize the error performance. Let $v_{l}$ denote the randomization (or time-sharing) factor for detector $\phi_{l}^{(k)}$, where $\sum_{l=1}^{N_{\mathrm{d}}} v_{l}=1$ and $v_{l} \geq 0$ for $l=1, \ldots, N_{\mathrm{d}}$. In other words, user $k$ employs detector $\phi_{l}^{(k)}$ for $100 v_{l}$ percent of the time, where $l \in\left\{1, \ldots, N_{\mathrm{d}}\right\}$ and $k \in\{1, \ldots, K\} .{ }^{4}$ It should be noted that employing the same randomization factors for all users does not cause any loss of generality since the cases in which different randomization factors are used for different users can be covered by the preceding formulation by considering an updated value of $N_{\mathrm{d}}$ with corresponding detectors and randomization factors.

\section{Optimal Detector RANDOMIZATION}

The aim in this study is to jointly optimize the randomization factors, the detectors (decision regions), and the transmitted signal amplitudes for all the users under an average power constraint. In order to formulate this generic problem, we first define the following signal vector $\boldsymbol{S}_{l}$ that consists of the signal amplitudes intended for detector $l$ for bit 0 and bit

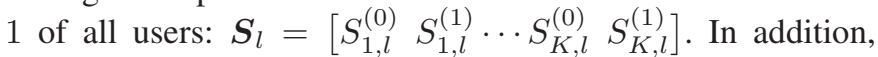
let $\phi_{l}$ denote the set of the $l^{\text {th }}$ detectors of the users, which is defined as $\phi_{l}=\left[\phi_{l}^{(1)} \cdots \phi_{l}^{(K)}\right]$ for $l \in\left\{1, \ldots, N_{\mathrm{d}}\right\}$. For a randomization strategy specified by randomization factors $\left\{v_{1}, \ldots, v_{N_{\mathrm{d}}}\right\}$ (as described in the previous paragraph), the system in Fig. 1 operates as follows: For $v_{l}$ fraction of the time, the transmitter sends the signal vector $\boldsymbol{S}_{l}$ and the users

\footnotetext{
${ }^{4}$ It is assumed that statistics of channel noise do not change during this randomization (time-sharing) operation. Therefore, the detector randomization approach is well-suited for block fading channels, where detector randomization can be performed for each channel realization [34].
} 
employ the corresponding detectors in $\phi_{l}$ for $l=1, \ldots, N_{\mathrm{d}}$. Therefore, the aim is to obtain the optimal set $\left\{v_{l}, \boldsymbol{\phi}_{l}, \boldsymbol{S}_{l}\right\}_{l=1}^{N_{\mathrm{d}}}$ that optimizes the error performance of the system under an average power constraint. Specifically, the following optimization problem is proposed:

$$
\begin{aligned}
\min _{\left\{v_{l}, \boldsymbol{\phi}_{l}, \boldsymbol{S}_{l}\right\}_{l=1}^{N_{\mathrm{d}}}} \max _{k \in\{1, \ldots, K\}} \mathrm{P}_{k} \\
\text { subject to } \mathrm{E}\left\{\int|p(t)|^{2} d t\right\} \leq A
\end{aligned}
$$

where $\mathrm{P}_{k}$ is the average probability of error for user $k$, $A$ specifies an average power constraint, and $p(t)$ is as in (1). Similar to [5], the minimax approach is adopted for fairness [35]-[38] by preventing scenarios in which the average probabilities of error are very low for some users whereas they are (unacceptably) high for others. ${ }^{5}$

The constraint in (6) is defined in such a way that the average power is limited in each bit duration. In other words, the expectation operation in (6) is over the equiprobable information bits of the users. Hence, from (1), (6) can be expressed as

$$
\sum_{k=1}^{K} \sum_{j=1}^{K} \rho_{k, j} \mathrm{E}\left\{S_{k, l}^{\left(i_{k}\right)} S_{j, l}^{\left(i_{j}\right)}\right\} \leq A,
$$

where $\mathrm{E}\left\{S_{k, l}^{\left(i_{k}\right)} S_{j, l}^{\left(i_{j}\right)}\right\}$ is given by

$\mathrm{E}\left\{S_{k, l}^{\left(i_{k}\right)} S_{j, l}^{\left(i_{j}\right)}\right\}=\left\{\begin{array}{cc}0.25 S_{k, l}^{(0)} S_{j, l}^{(0)}+0.25 S_{k, l}^{(0)} S_{j, l}^{(1)} & \\ +0.25 S_{k, l}^{(1)} S_{j, l}^{(0)}+0.25 S_{k, l}^{(1)} S_{j, l}^{(1)}, & k \neq j \\ 0.5\left|S_{k, l}^{(0)}\right|^{2}+0.5\left|S_{k, l}^{(1)}\right|^{2}, & k=j\end{array}\right.$

for $l \in\left\{1, \ldots, N_{\mathrm{d}}\right\}$. If symmetric signaling is employed, (i.e., if signal amplitudes are selected as $S_{k, l}^{(0)}=-S_{k, l}^{(1)}$ for $k=$ $1, \ldots, K$ and $l=1, \ldots, N_{\mathrm{d}}$ ), then $\mathrm{E}\left\{S_{k, l}^{\left(i_{k}\right)} S_{j, l}^{\left(i_{j}\right)}\right\}=\left|S_{k, l}^{(1)}\right|^{2}$ for $k=j$ and $\mathrm{E}\left\{S_{k, l}^{\left(i_{k}\right)} S_{j, l}^{\left(i_{j}\right)}\right\}=0$ for $k \neq j$. Then, the expression in (7) becomes $\sum_{k=1}^{K}\left|S_{k, l}^{(1)}\right|^{2} \leq A$. (We consider the generic case in this study and the results for symmetric signaling can be obtained as a special case.)

For notational simplicity in the following analysis, we define

$$
h\left(\boldsymbol{S}_{l}\right) \triangleq \sum_{k=1}^{K} \sum_{j=1}^{K} \rho_{k, j} \mathrm{E}\left\{S_{k, l}^{\left(i_{k}\right)} S_{j, l}^{\left(i_{j}\right)}\right\}
$$

where $S_{l}$ is as defined in the first paragraph of this section. Then, the average power constraint in (7) (hence, in (6)) is given by

$$
h\left(\boldsymbol{S}_{l}\right) \leq A \text { for } l \in\left\{1, \ldots, N_{\mathrm{d}}\right\} .
$$

In order to calculate the average probability of error for user $k, \mathrm{P}_{k}$, we first express, from (3) and (4), the error probability of the $l^{\text {th }}$ detector of user $k$ when the signal vector $\boldsymbol{S}_{l}$ is

\footnotetext{
${ }^{5}$ It is possible to extend the results to cases in which different users have different levels of importance by multiplying each $\mathrm{P}_{k}$ with a weighting factor.
}

employed as follows:

$$
\begin{aligned}
& g_{k, l}\left(\boldsymbol{S}_{l}\right)= \\
& \frac{1}{2^{K}} \sum_{\mathbf{i}_{k} \in\{0,1\}^{K-1}}\left(\mathrm{P}\left\{\left(N_{k}+S_{k, l}^{(1)}+\sum_{\substack{j=1 \\
j \neq k}}^{K} \rho_{k, j} S_{j, l}^{\left(i_{j}\right)}\right) \notin \Gamma_{l}^{(k)}\right\}\right. \\
& \left.\quad+\mathrm{P}\left\{\left(N_{k}+S_{k, l}^{(0)}+\sum_{\substack{j=1 \\
j \neq k}}^{K} \rho_{k, j} S_{j, l}^{\left(i_{j}\right)}\right) \in \Gamma_{l}^{(k)}\right\}\right),
\end{aligned}
$$

with $\mathbf{i}_{k} \triangleq\left[\begin{array}{lll}i_{1} & \cdots i_{k-1} & i_{k+1} \cdots i_{K}\end{array}\right]$ (the vector of all the bit indices except for the $k^{\text {th }}$ one), and $\Gamma_{l}^{(k)}$ denoting the decision region of the $l^{\text {th }}$ detector of user $k$ for information symbol 1 ; that is, $\phi_{l}^{(k)}$, as specified in (4). In (11), the probabilities are with respect to the distribution of the noise component $N_{k}$ for a given value of $\boldsymbol{S}_{l}$. Also, it should be noted that the decision region $\Gamma_{l}^{(k)}$ can be a function of $\boldsymbol{S}_{l}$ in general due to the joint optimization in (5) and (6).

Since $g_{k, l}\left(\boldsymbol{S}_{l}\right)$ in (11) denotes the error probability of the $l^{\text {th }}$ detector of user $k$ when signal vector $\boldsymbol{S}_{l}$ is employed, the average probability of user $k$ for a randomization strategy that employs signal vector $\boldsymbol{S}_{l}$ and detectors $\phi_{l}$ with probability $v_{l}$ for $l=1, \ldots, N_{\mathrm{d}}$ can be expressed as

$$
\mathrm{P}_{k}=\sum_{l=1}^{N_{\mathrm{d}}} v_{l} g_{k, l}\left(\boldsymbol{S}_{l}\right)
$$

From (10) and (12), the optimization problem in (5) and (6) can be stated as

$$
\begin{aligned}
\min _{\left\{v_{l}, \boldsymbol{\phi}_{l}, \boldsymbol{S}_{l}\right\}_{l=1}^{N_{\mathrm{d}}}} & \max _{k \in\{1, \ldots, K\}} \sum_{l=1}^{N_{\mathrm{d}}} v_{l} g_{k, l}\left(\boldsymbol{S}_{l}\right) \\
\text { subject to } & h\left(\boldsymbol{S}_{l}\right) \leq A, \forall l \in\left\{1, \ldots, N_{\mathrm{d}}\right\} \\
& \sum_{l=1}^{N_{\mathrm{d}}} v_{l}=1, v_{l} \geq 0, \forall l \in\left\{1, \ldots, N_{\mathrm{d}}\right\} .
\end{aligned}
$$

This problem is very challenging in general since it requires joint optimization of the signal amplitudes, the detectors, and the detector randomization factors. However, a significant simplification can be achieved based on the following proposition:

Proposition 1: The optimization problem in (13)-(15) can be expressed as

$\min _{\left\{v_{l}, \boldsymbol{S}_{l}\right\}_{l=1}^{N_{\mathrm{d}}}} \max _{k \in\{1, \ldots, K\}} \sum_{l=1}^{N_{\mathrm{d}}} \frac{v_{l}}{2} \int_{-\infty}^{\infty} \min \left\{p_{0}^{(k)}\left(y \mid \boldsymbol{S}_{l}\right), p_{1}^{(k)}\left(y \mid \boldsymbol{S}_{l}\right)\right\} d y$

subject to $h\left(\boldsymbol{S}_{l}\right) \leq A, \forall l \in\left\{1, \ldots, N_{\mathrm{d}}\right\}$

$$
\sum_{l=1}^{N_{\mathrm{d}}} v_{l}=1, v_{l} \geq 0, \forall l \in\left\{1, \ldots, N_{\mathrm{d}}\right\}
$$


where $p_{i_{k}}^{(k)}\left(y \mid \boldsymbol{S}_{l}\right)$ is given by

$$
\begin{aligned}
& p_{i_{k}}^{(k)}\left(y \mid \boldsymbol{S}_{l}\right)=\frac{1}{\sigma_{k} \sqrt{2 \pi} 2^{K-1}} \\
& \times \sum_{\mathbf{i}_{k} \in\{0,1\}^{K-1}} \exp \left\{-\frac{1}{2 \sigma_{k}^{2}}\left(y-S_{k, l}^{\left(i_{k}\right)}-\sum_{\substack{j=1 \\
j \neq k}}^{K} \rho_{k, j} S_{j, l}^{\left(i_{j}\right)}\right)^{2}\right\}
\end{aligned}
$$

for $i_{k}=0,1$ with $\mathbf{i}_{k} \triangleq\left[i_{1} \cdots i_{k-1} i_{k+1} \cdots i_{K}\right]$.

Proof: Consider the optimization problem in (13)-(15), where $g_{k, l}\left(\boldsymbol{S}_{l}\right)$ is defined as in (11) and represents the error probability of the $l^{\text {th }}$ detector of user $k$ when signal vector $S_{l}$ is employed. Since the aim is to minimize $\max _{k \in\{1, \ldots, K\}} \sum_{l=1}^{N_{\mathrm{d}}} v_{l} g_{k, l}\left(\boldsymbol{S}_{l}\right)$ over all possible $\left\{v_{l}, \boldsymbol{\phi}_{l}, \boldsymbol{S}_{l}\right\}_{l=1}^{N_{\mathrm{d}}}$ under the specified constraints, optimal decision rules, $\phi_{l}$, that minimize $g_{k, l}\left(\boldsymbol{S}_{l}\right)$ must be employed for each signal vector $\boldsymbol{S}_{l}$. For any signal vector, it is known that the ML detector minimizes the error probability when the information symbols are equally likely [39]. Therefore, it is concluded that the optimal solution to (13)-(15) results in the use of ML detectors at the receivers. Considering the $l^{\text {th }}$ detector of user $k$, the ML decision rule can be specified as $i_{k}=1$ if $p_{1}^{(k)}\left(y \mid \boldsymbol{S}_{l}\right) \geq p_{0}^{(k)}\left(y \mid \boldsymbol{S}_{l}\right)$ and $i_{k}=0$ otherwise, where $p_{i_{k}}^{(k)}\left(y \mid \boldsymbol{S}_{l}\right)$ is the conditional probability density function (PDF) of observation $Y_{k}$ when the information bit $i_{k}$ is transmitted for the $l^{\text {th }}$ detector of user $k$ (see (3)). Therefore, the error probability of the ML detector can be calculated from $\frac{1}{2} \int \min \left\{p_{0}^{(k)}\left(y \mid \boldsymbol{S}_{l}\right), p_{1}^{(k)}\left(y \mid \boldsymbol{S}_{l}\right)\right\} d y$ [1], which corresponds to $g_{k, l}\left(\boldsymbol{S}_{l}\right)$ when the $l^{\text {th }}$ detector of user $k$ employs the ML decision rule. Hence, the expression in (16) is obtained from (13). (It is noted that the optimization space is reduced from $\left\{v_{l}, \phi_{l}, \boldsymbol{S}_{l}\right\}_{l=1}^{N_{\mathrm{d}}}$ to $\left\{v_{l}, \boldsymbol{S}_{l}\right\}_{l=1}^{N_{\mathrm{d}}}$ since the error probabilities of the optimal detectors are expressed in terms of the signal vectors.) In addition, based on (3), $p_{i_{k}}^{(k)}\left(y \mid \boldsymbol{S}_{l}\right)$ can be expressed as in (19) considering equally likely information bits.

Based on Proposition 1, it is concluded that for the joint optimization problem in (13)-(15), where the detectors are modeled as generic ones, the joint optimal solution always results in the use of $\mathrm{ML}$ detectors at all the users. It is also noted that the results of Proposition 1 will be valid for any non-Gaussian PDF as well when the conditional PDF expression in (19) is updated accordingly.

Comparison of the optimization problems in (13)-(15) and in (16)-(18) reveals that Proposition 1 provides a significant simplification in obtaining the optimal solution as it reduces the optimization space from $\left\{v_{l}, \boldsymbol{\phi}_{l}, \boldsymbol{S}_{l}\right\}_{l=1}^{N_{\mathrm{d}}}$ to $\left\{v_{l}, \boldsymbol{S}_{l}\right\}_{l=1}^{N_{\mathrm{d}}}$. Namely, instead of searching over all possible signal amplitudes, detectors, and detector randomization factors, (16)-(18) requires a search over possible signal amplitudes and detector randomization factors. Once the optimal signal amplitudes and detector randomization factors are obtained from (16)-(18), the optimal detectors are specified by the corresponding ML decision rules. In particular, if $\left\{\hat{\boldsymbol{S}}_{l}\right\}_{l=1}^{N_{\mathrm{d}}}$ denote the optimal signal amplitudes obtained from (16)-(18), the $l^{\text {th }}$ detector of user $k$ outputs bit 1 if $p_{1}^{(k)}\left(y \mid \hat{\boldsymbol{S}}_{l}\right) \geq p_{0}^{(k)}\left(y \mid \hat{\boldsymbol{S}}_{l}\right)$ and bit 0 otherwise for $k \in\{1, \ldots, K\}$ and $l \in\left\{1, \ldots, N_{\mathrm{d}}\right\}$, where $p_{0}^{(k)}\left(y \mid \hat{\boldsymbol{S}}_{l}\right)$ and $p_{1}^{(k)}\left(y \mid \hat{\boldsymbol{S}}_{l}\right)$ are obtained from (19).

Although the formulation in (16)-(18) provides a signif- icant simplification over that in (13)-(15), it can still have high computational complexity when the number of detectors and/or the number of users are high. In particular, it is noted from (16)-(18) that the optimal solution of the signal amplitudes and the randomization factors requires a search over a $(2 K+1) N_{\mathrm{d}}$ dimensional space $\left((K+1) N_{\mathrm{d}}\right.$ dimensional space if symmetric signaling is employed). In the following proposition, it is stated that employing more than $K$ detectors at a receiver is not needed for the optimal solution.

Proposition 2: The optimization problem in (16)-(18) achieves the same minimum value as the following problem:

$$
\begin{aligned}
\min _{\left\{v_{l}, \boldsymbol{S}_{l}\right\}_{l=1}^{\min \left\{K, N_{\mathrm{d}}\right\}}} \max _{k \in\{1, \ldots, K\}} \sum_{l=1}^{\min \left\{K, N_{\mathrm{d}}\right\}} \frac{v_{l}}{2} \\
\times \int_{-\infty}^{\infty} \min \left\{p_{0}^{(k)}\left(y \mid \boldsymbol{S}_{l}\right), p_{1}^{(k)}\left(y \mid \boldsymbol{S}_{l}\right)\right\} d y
\end{aligned}
$$

subject to $h\left(\boldsymbol{S}_{l}\right) \leq A, \forall l \in\left\{1, \ldots, \min \left\{K, N_{\mathrm{d}}\right\}\right\}$

$$
\sum_{l=1}^{\min \left\{K, N_{\mathrm{d}}\right\}} v_{l}=1, v_{l} \geq 0, \forall l \in\left\{1, \ldots, \min \left\{K, N_{\mathrm{d}}\right\}\right\}
$$

where $p_{i_{k}}^{(k)}\left(y \mid \boldsymbol{S}_{l}\right)$ is as in (19).

Proof: Define

$$
\tilde{g}_{k}\left(\boldsymbol{S}_{l}\right) \triangleq 0.5 \int_{-\infty}^{\infty} \min \left\{p_{0}^{(k)}\left(y \mid \boldsymbol{S}_{l}\right), p_{1}^{(k)}\left(y \mid \boldsymbol{S}_{l}\right)\right\} d y
$$

and express the objective function in (16) as $\sum_{l=1}^{N_{\mathrm{d}}} v_{l} \tilde{g}_{k}\left(\boldsymbol{S}_{l}\right)=$ $\mathrm{E}\left\{\tilde{g}_{k}(\boldsymbol{S})\right\}$, where $\boldsymbol{S}$ is a discrete random vector that takes the value of $\boldsymbol{S}_{l}$ with probability $v_{l}$ for $l=1, \ldots, N_{\mathrm{d}}$ (cf. (18)). Let $p_{\boldsymbol{S}}$ denote the probability mass function (PMF) of $\boldsymbol{S}$. In addition, define $\mathcal{P}_{A}$ as the set of all PMFs with $N_{\mathrm{d}}$ point masses for which $p_{\boldsymbol{S}}(\boldsymbol{S})=0$ whenever $h(\boldsymbol{S})>A$. Then, (16)-(18) can be expressed as

$$
\min _{p_{\boldsymbol{S}} \in \mathcal{P}_{A}} \max _{k \in\{1, \ldots, K\}} \mathrm{E}\left\{\tilde{g}_{k}(\boldsymbol{S})\right\} .
$$

Optimization problems that are in similar forms to (24) have been studied in the literature, such as in [12] and [11]. First, the following set is defined: $U=\left\{\left(\tilde{g}_{1}(\boldsymbol{S}), \ldots, \tilde{g}_{K}(\boldsymbol{S})\right), \forall \boldsymbol{S} \in\right.$ $\mathcal{S}_{A}$ \}, where $\mathcal{S}_{A}$ is the set of $\boldsymbol{S}$ for which $h(\boldsymbol{S}) \leq A$. Then, it can be observed that set $W$, defined as $W=$ $\left\{\left(\mathrm{E}\left\{\tilde{g}_{1}(\boldsymbol{S})\right\}, \ldots, \mathrm{E}\left\{\tilde{g}_{K}(\boldsymbol{S})\right\}\right), \forall p_{\boldsymbol{S}} \in \mathcal{P}_{A}\right\}$, corresponds to the convex hull of set $U$. Therefore, based on Carathéodory's theorem [40], any $K$-tuple at the boundary of set $W$ can be obtained as the convex combination of at most $K$ elements in $U$. (The boundary is considered since a minimization operation is to performed.) Hence, the optimal solution to (24) can be expressed in the form of a discrete random vector with at most $K$ non-zero point masses. For this reason, if $N_{\mathrm{d}}$ is larger than $K$, it is sufficient to perform the search over probability distributions with $K$ point masses.

Based on Proposition 2, it is concluded that there is no need for employing more than $K$ detectors at a receiver in a $K$-user system for achieving the optimal error performance. In other words, randomization among more than $K$ detectors cannot provide any additional performance improvements. In addition, as observed from (20)-(22), the dimension of the search space in obtaining the optimal solution is specified by $(2 K+1) \min \left\{K, N_{\mathrm{d}}\right\}$ (by $(K+1) \min \left\{K, N_{\mathrm{d}}\right\}$ for symmetric 
signaling). It is also noted that the results of Proposition 2 will be valid for non-Gaussian PDFs as well when the conditional PDF expression in (19) is updated accordingly.

\section{ANALYSis of Optimal Detector RANDOMization}

In this section, we investigate the performance of the optimal detector randomization approach specified by (20)-(22), and determine scenarios in which performance improvements can be obtained over the optimal approach that does not employ any detector randomization, which is called as the optimal single detectors approach in the following.

The optimal single detectors approach can be considered as a special case of the detector randomization approach when there is only one detector at each receiver; that is, $N_{\mathrm{d}}=1$. Therefore, based on (13)-(15), the optimal single detectors approach can be specified by the following optimization problem:

$$
\begin{aligned}
& \min _{\boldsymbol{\phi}, \boldsymbol{S}} \max _{\begin{array}{c}
k \in\{1, \ldots, K\} \\
\text { subject to }
\end{array}} g_{k}(\boldsymbol{S}) \leq A \\
& \text { s }
\end{aligned}
$$

where $g_{k}(\boldsymbol{S})$ can be expressed as in (11) by removing the dependence on $l$ in the expressions (since there is only one detector for each user), $\phi=\left[\phi^{(1)} \cdots \phi^{(K)}\right]$ represents the detectors of the users, and $\boldsymbol{S}$ is the vector of signal amplitudes for bit 0 and bit 1 of all users; i.e., $\boldsymbol{S}=\left[S_{1}^{(0)} S_{1}^{(1)} \cdots S_{K}^{(0)} S_{K}^{(1)}\right]$.

Since (25) is a special case of (13)-(15), its solution can be obtained from Proposition 1 by setting $N_{\mathrm{d}}=1$ in (16)(18). Hence, the optimal single detectors approach can also be formulated as

$$
\begin{aligned}
& \min _{\boldsymbol{S}} \max _{k \in\{1, \ldots, K\}} \tilde{g}_{k}(\boldsymbol{S}) \\
& \text { subject to } h(\boldsymbol{S}) \leq A
\end{aligned}
$$

where $\tilde{g}_{k}(\boldsymbol{S})$ is as defined in (23). In other words, the optimal single detectors approach requires the calculation of the optimal signal amplitudes from (26). Then, each user employs the corresponding ML detector, which selects bit 1 if $p_{1}^{(k)}\left(y \mid \boldsymbol{S}^{\diamond}\right) \geq p_{0}^{(k)}\left(y \mid \boldsymbol{S}^{\diamond}\right)$ and bit 0 otherwise, where $\boldsymbol{S}^{\diamond}$ denotes the solution of (26).

Let $\mathrm{P}_{\mathrm{SD}}$ denote the optimal value achieved by the optimization problem in (26) (equivalently, (25)); that is, the minimum worst-case (maximum) average probability of error corresponding to the optimal single detectors approach. Similarly, let $\mathrm{P}_{\mathrm{DR}}$ represent the solution of the optimization problem in (20)-(22) (equivalently, (13)-(15)), which is the minimum worst-case average probability of error achieved by the optimal detector randomization approach. The main purpose of this section is to provide bounds on $\mathrm{P}_{\mathrm{DR}}$, and to specify various relations between $\mathrm{P}_{\mathrm{SD}}$ and $\mathrm{P}_{\mathrm{DR}}$. First, the following proposition is obtained to provide a lower bound on $\mathrm{P}_{\mathrm{DR}}$.

Proposition 3: The minimum worst-case average probability of error achieved by the optimal detector randomization approach in (20)-(22), $\mathrm{P}_{\mathrm{DR}}$, is lower bounded as follows:

$$
\mathrm{P}_{\mathrm{DR}} \geq \frac{1}{K} \sum_{k=1}^{K} \tilde{g}_{k}\left(\boldsymbol{S}^{*}\right) \triangleq \mathrm{P}_{\mathrm{LB}}
$$

with

$$
\boldsymbol{S}^{*}=\arg \min _{\boldsymbol{S} \in \mathcal{S}_{A}} \sum_{k=1}^{K} \tilde{g}_{k}(\boldsymbol{S})
$$

where $\mathcal{S}_{A}$ is defined as $\mathcal{S}_{A} \triangleq\{\boldsymbol{S}: h(\boldsymbol{S}) \leq A\}$ and $\tilde{g}_{k}(\boldsymbol{S})$ is as in (23). In addition, the lower bound in (27) is achieved; that is, $\mathrm{P}_{\mathrm{DR}}=\mathrm{P}_{\mathrm{LB}}$, if and only if there exists feasible $\left\{v_{l}, \boldsymbol{S}_{l}\right\}_{l=1}^{\min \left\{K, N_{\mathrm{d}}\right\}}$ (i.e., satisfying (21) and (22)) such that $\sum_{l=1}^{\min \left\{K, N_{\mathrm{d}}\right\}} v_{l} \tilde{g}_{k}\left(\boldsymbol{S}_{l}\right)=\mathrm{P}_{\mathrm{LB}}, \forall k \in\{1, \ldots, K\}$.

Proof: Consider a modified version of the optimization problem in (20)-(22), which is described as

$$
\begin{aligned}
& \min _{\left\{v_{l}, \boldsymbol{S}_{l}\right\}_{l=1}^{\min \left\{K, N_{\mathrm{d}}\right\}}} \frac{1}{K} \sum_{k=1}^{K} \sum_{l=1}^{\min \left\{K, N_{\mathrm{d}}\right\}} v_{l} \tilde{g}_{k}\left(\boldsymbol{S}_{l}\right) \\
& \text { subject to } h\left(\boldsymbol{S}_{l}\right) \leq A, \forall l \in\left\{1, \ldots, \min \left\{K, N_{\mathrm{d}}\right\}\right\} \\
& \sum_{l=1}^{\min \left\{K, N_{\mathrm{d}}\right\}} v_{l}=1, v_{l} \geq 0, \forall l \in\left\{1, \ldots, \min \left\{K, N_{\mathrm{d}}\right\}\right\}
\end{aligned}
$$

where $\tilde{g}_{k}\left(\boldsymbol{S}_{l}\right)$ is given by (23). Define $g_{\mathrm{avg}}(\boldsymbol{S}) \triangleq$ $\frac{1}{K} \sum_{k=1}^{K} \tilde{g}_{k}(\boldsymbol{S})$ and express the problem in (29)-(31) as

$$
\begin{aligned}
& \min _{\left\{v_{l}, \boldsymbol{S}_{l} \in \mathcal{S}_{A}\right\}_{l=1}^{\min \left\{K, N_{\mathrm{d}}\right\}}} \sum_{l=1}^{\min \left\{K, N_{\mathrm{d}}\right\}} v_{l} g_{\mathrm{avg}}\left(\boldsymbol{S}_{l}\right) \\
& \text { subject to } \sum_{l=1}^{\min \left\{K, N_{\mathrm{d}}\right\}} v_{l}=1, v_{l} \geq 0, \forall l \in\left\{1, \ldots, \min \left\{K, N_{\mathrm{d}}\right\}\right\}
\end{aligned}
$$

where $\mathcal{S}_{A}$ is as described in the proposition. The optimal solution of (32)-(33) is obtained by assigning all the weight to the minimizer of $g_{\mathrm{avg}}(\boldsymbol{S})$ over $\mathcal{S}_{A}$, which corresponds to $\boldsymbol{S}^{*}$ defined in (28). For example, $v_{1}=1, v_{l}=0$ for $l=2, \ldots, N_{\mathrm{d}}$, and $\boldsymbol{S}_{1}=\boldsymbol{S}^{*}$ achieves the minimum value of the objective function in (32)-(33). Therefore, the minimum value achieved by the optimization problem in (29)-(31) is equal to $g_{\text {avg }}\left(\boldsymbol{S}^{*}\right)=\frac{1}{K} \sum_{k=1}^{K} \tilde{g}_{k}\left(\boldsymbol{S}^{*}\right)$. When the optimization problems in (20)-(22) and in (29)-(31) are compared, it is observed that the latter provides a lower bound on the former since the average of the error probabilities of the users is considered in (29) whereas the maximum of the error probabilities is employed in (20). (Please note the $\frac{1}{K} \sum_{k=1}^{K}$ and $\max _{k \in\{1, \ldots, K\}}$ operators, respectively.) Therefore, the solution of (29)-(31), which is specified by $\frac{1}{K} \sum_{k=1}^{K} \tilde{g}_{k}\left(\boldsymbol{S}^{*}\right)$, provides a lower bound on the solution of (20)-(22), $\mathrm{P}_{\mathrm{DR}}$. Hence, (27) is obtained.

In order to prove the sufficiency of the achievability condition in Proposition 3, assume that there exists feasible $\left\{v_{l}, \boldsymbol{S}_{l}\right\}_{l=1}^{\min \left\{K, N_{\mathrm{d}}\right\}}$ (i.e., satisfying (21) and (22)) such that $\sum_{l=1}^{\min \left\{K, N_{\mathrm{d}}\right\}} v_{l} \tilde{g}_{k}\left(\boldsymbol{S}_{l}\right)=\mathrm{P}_{\mathrm{LB}}, \forall k \in\{1, \ldots, K\}$. Then, it is easy to verify from (20) and (23) that the summation term in (20) becomes equal to $\mathrm{P}_{\mathrm{LB}}, \forall k \in\{1, \ldots, K\}$, for the specified solution. Hence, (20)-(22) achieves the lower bound in this case, and $\mathrm{P}_{\mathrm{DR}}=\mathrm{P}_{\mathrm{LB}}$ is obtained. For proving the necessity of the achievability condition in the proposition via contradiction, assume that $\mathrm{P}_{\mathrm{DR}}=\mathrm{P}_{\mathrm{LB}}$ and the optimal solution of (20)-(22), denoted by $\left\{\hat{v}_{l}, \hat{\boldsymbol{S}}_{l}\right\}_{l=1}^{\min \left\{K, N_{\mathrm{d}}\right\}}$, results in a scenario in which the $\sum_{l=1}^{\min \left\{K, N_{\mathrm{d}}\right\}} \hat{v}_{l} \tilde{g}_{k}\left(\hat{\boldsymbol{S}}_{l}\right)$ 
terms are not all the same. In particular, assume that $\exists k^{\prime} \in$ $\{1, \ldots, K\}$ such that $\sum_{l=1}^{\min \left\{K, N_{\mathrm{d}}\right\}} \hat{v}_{l} \tilde{g}_{k^{\prime}}\left(\hat{\boldsymbol{S}}_{l}\right)<\mathrm{P}_{\mathrm{LB}}$ and that $\sum_{l=1}^{\min \left\{K, N_{\mathrm{d}}\right\}} \hat{v}_{l} \tilde{g}_{k}\left(\hat{\boldsymbol{S}}_{l}\right)=\mathrm{P}_{\mathrm{LB}}, \forall k \in\{1, \ldots, K\} \backslash\left\{k^{\prime}\right\}{ }^{6}$ Then, the following inequality is obtained:

$$
\frac{1}{K} \sum_{k=1}^{K} \sum_{l=1}^{\min \left\{K, N_{\mathrm{d}}\right\}} \hat{v}_{l} \tilde{g}_{k}\left(\hat{\boldsymbol{S}}_{l}\right)<\mathrm{P}_{\mathrm{LB}} \text {. }
$$

However, this implies a contradiction since

$$
\begin{aligned}
& \frac{1}{K} \sum_{k=1}^{K} \sum_{l=1}^{\min \left\{K, N_{\mathrm{d}}\right\}} \hat{v}_{l} \tilde{g}_{k}\left(\hat{\boldsymbol{S}}_{l}\right) \\
& =\sum_{l=1}^{\min \left\{K, N_{\mathrm{d}}\right\}} \hat{v}_{l}\left(\frac{1}{K} \sum_{k=1}^{K} \tilde{g}_{k}\left(\hat{\boldsymbol{S}}_{l}\right)\right) \geq \mathrm{P}_{\mathrm{LB}}
\end{aligned}
$$

where the inequality follows from (27). Therefore, when the lower bound is achieved, i.e., $\mathrm{P}_{\mathrm{DR}}=\mathrm{P}_{\mathrm{LB}}$, all the $\sum_{l=1}^{\min \left\{K, N_{\mathrm{d}}\right\}} \hat{v}_{l} \tilde{g}_{k}\left(\hat{\boldsymbol{S}}_{l}\right)$ terms must be equal to $\mathrm{P}_{\mathrm{LB}}$. Hence, in order to achieve the lower bound in (27), there must exist feasible $\left\{v_{l}, \boldsymbol{S}_{l}\right\}_{l=1}^{\min \left\{K, N_{\mathrm{d}}\right\}}$ such that $\sum_{l=1}^{\min \left\{K, N_{\mathrm{d}}\right\}} v_{l} \tilde{g}_{k}\left(\boldsymbol{S}_{l}\right)=$ $\mathrm{P}_{\mathrm{LB}}, \forall k \in\{1, \ldots, K\}$, as stated in the proposition.

Proposition 3 presents a bound on the performance of the optimal detector randomization approach in (20)-(22). The advantage of this lower bound is that it is calculated based on the solution of the minimization problem in (28), which is much simpler than the optimization problem in (20)-(22). In addition, the achievability condition in Proposition 3 implies that the worst-case average probability of error achieved by the optimal detector randomization approach attains the lower bound if and only if there exists an equalizer solution for the optimal detector randomization problem in (20)-(22), which equates the average error probabilities of all users to the lower bound in (27). As a simple example, if $S^{*}$ in (28) satisfies that $\tilde{g}_{1}\left(\boldsymbol{S}^{*}\right)=\cdots=\tilde{g}_{K}\left(\boldsymbol{S}^{*}\right)$, then $v_{1}=1$, $v_{l}=0$ for $l=2, \ldots, \min \left\{K, N_{\mathrm{d}}\right\}$, and $\boldsymbol{S}_{1}=\boldsymbol{S}^{*}$ results in $\sum_{l=1}^{\min \left\{K, N_{\mathrm{d}}\right\}} v_{l} \tilde{g}_{k}\left(\boldsymbol{S}_{l}\right)=\tilde{g}_{k}\left(\boldsymbol{S}^{*}\right)=\mathrm{P}_{\mathrm{LB}}, \forall k \in\{1, \ldots, K\}$; hence, the lower bound is achieved in this scenario; i.e., $\mathrm{P}_{\mathrm{DR}}=\mathrm{P}_{\mathrm{LB}}$, as a result of Proposition 3. As investigated in the following, there also exist other scenarios in which $\mathrm{P}_{\mathrm{DR}}=\mathrm{P}_{\mathrm{LB}}$ is satisfied when all $\tilde{g}_{k}\left(\boldsymbol{S}^{*}\right)$ 's are not the same.

Next, improvements that can be achieved via the optimal detector randomization approach over the optimal single detectors approach are quantified in the following proposition.

Proposition 4: Let $\mathrm{P}_{\mathrm{SD}}$ and $\mathrm{P}_{\mathrm{DR}}$ denote the minimum worst-case error probabilities obtained from the solutions of (26) and (20)-(22), respectively. Then, the following relations hold between $\mathrm{P}_{\mathrm{SD}}$ and $\mathrm{P}_{\mathrm{DR}}$.

(i) The improvement ratio, defined as $\mathrm{P}_{\mathrm{SD}} / \mathrm{P}_{\mathrm{DR}}$, is bounded as follows:

$$
1 \leq \frac{\mathrm{P}_{\mathrm{SD}}}{\mathrm{P}_{\mathrm{DR}}} \leq K
$$

(ii) The maximum improvement ratio, $K$, is achieved if and only if $\mathrm{P}_{\mathrm{DR}}=\mathrm{P}_{\mathrm{LB}}$ (where $\mathrm{P}_{\mathrm{LB}}$ is as defined in (27)),

\footnotetext{
${ }^{6}$ Note that none of the $\sum_{l=1}^{\min \left\{K, N_{\mathrm{d}}\right\}} \hat{v}_{l} \tilde{g}_{k}\left(\hat{\boldsymbol{S}}_{l}\right)$ terms can be larger than $\mathrm{P}_{\mathrm{LB}}$ since it is assumed that $\mathrm{P}_{\mathrm{DR}}=\mathrm{P}_{\mathrm{LB}}$; i.e., the maximum of these terms is equal to $\mathrm{P}_{\mathrm{LB}}$ (see (20) and (23)). Therefore, either all these terms can be equal to $\mathrm{P}_{\mathrm{LB}}$ or some of them can be smaller than $\mathrm{P}_{\mathrm{LB}}$. The latter is shown to be impossible in the remaining part of the proof.
}

and $S^{*}$ in (28) is the optimal solution to the optimization problem in (26) with $\tilde{g}_{k}\left(\boldsymbol{S}^{*}\right)=0, \forall k \in\{1, \ldots, K\} \backslash$ $\left\{k^{*}\right\}$ and $\tilde{g}_{k^{*}}\left(\boldsymbol{S}^{*}\right)>0$, where $\tilde{g}_{k}$ is given by (23) and $k^{*}$ is any value in $\{1, \ldots, K\}$.

(iii) No improvement is achieved; that is, $\mathrm{P}_{\mathrm{DR}}=\mathrm{P}_{\mathrm{SD}}$, if $\tilde{g}_{1}\left(\boldsymbol{S}^{*}\right)=\cdots=\tilde{g}_{K}\left(\boldsymbol{S}^{*}\right)$.

(iv) Improvement is guaranteed; that is, $\mathrm{P}_{\mathrm{DR}}<\mathrm{P}_{\mathrm{SD}}$, if $\mathrm{P}_{\mathrm{DR}}=\mathrm{P}_{\mathrm{LB}}$ and $\tilde{g}_{k}\left(\boldsymbol{S}^{\diamond}\right) \neq \tilde{g}_{l}\left(\boldsymbol{S}^{\diamond}\right)$ for some $k, l \in$ $\{1, \ldots, K\}$, where $\boldsymbol{S}^{\diamond}$ denotes the solution of (26).

Proof: (i) Since the optimal single detectors approach is a special case of the detector randomization approach, $\mathrm{P}_{\mathrm{DR}} \leq$ $\mathrm{P}_{\mathrm{SD}}$ is always satisfied; hence, the lower bound in (36) is directly obtained. In order to derive the upper bound in (36), the following inequalities are considered first:

$$
\mathrm{P}_{\mathrm{SD}}=\max _{k} \tilde{g}_{k}\left(\boldsymbol{S}^{\diamond}\right) \leq \max _{k} \tilde{g}_{k}\left(\boldsymbol{S}^{*}\right) \leq \sum_{k=1}^{K} \tilde{g}_{k}\left(\boldsymbol{S}^{*}\right)
$$

where $S^{\diamond}$ is the solution of (26), and $S^{*}$ is given by (28). Note that the first inequality follows by definition since $S^{\diamond}$ and $S^{*}$ are the solutions of (26) and (28), respectively, and the second inequality follows from the identity $\|\mathbf{x}\|_{\infty} \leq\|\mathbf{x}\|_{1}$, $\forall \mathbf{x}$, where $\|\mathbf{x}\|_{\infty}$ and $\|\mathbf{x}\|_{1}$ are the maximum and Manhattan norms, respectively. Then, the upper bound in (36) is obtained as follows:

$$
\frac{\mathrm{P}_{\mathrm{SD}}}{\mathrm{P}_{\mathrm{DR}}} \leq \frac{\sum_{k=1}^{K} \tilde{g}_{k}\left(\boldsymbol{S}^{*}\right)}{\mathrm{P}_{\mathrm{DR}}} \leq \frac{\sum_{k=1}^{K} \tilde{g}_{k}\left(\boldsymbol{S}^{*}\right)}{\mathrm{P}_{\mathrm{LB}}}=K
$$

where the first inequality is obtained from (37), and the second inequality and the equality follow from (27).

(ii) In order to achieve the maximum improvement ratio of $K$ in (36), the inequalities in (37) and (38) should hold with equality. Then, from (37), it is concluded that $S^{*}$ in (28) should also be a solution of (26) (so that $\max _{k} \tilde{g}_{k}\left(\boldsymbol{S}^{\diamond}\right)=$ $\max _{k} \tilde{g}_{k}\left(\boldsymbol{S}^{*}\right)$ ), and $\tilde{g}_{k}\left(\boldsymbol{S}^{*}\right)$ should be zero for all $k$ except for one of them (so that $\max \tilde{g}_{k}\left(\boldsymbol{S}^{*}\right)=\sum_{k=1}^{K} \tilde{g}_{k}\left(\boldsymbol{S}^{*}\right)$ ). In addition, for the second inequality in (38) to hold with equality, $\mathrm{P}_{\mathrm{DR}}=\mathrm{P}_{\mathrm{LB}}$ should be satisfied. Hence, the conditions in Part (ii) of Proposition 4 are obtained.

(iii) Consider a scenario in which $\tilde{g}_{1}\left(\boldsymbol{S}^{*}\right)=\cdots=\tilde{g}_{K}\left(\boldsymbol{S}^{*}\right)$. In order to prove that $\mathrm{P}_{\mathrm{DR}}=\mathrm{P}_{\mathrm{SD}}$ via contradiction, first suppose that $\max \tilde{g}_{k}\left(\boldsymbol{S}^{\diamond}\right)<\max \tilde{g}_{k}\left(\boldsymbol{S}^{*}\right)$. Then, the following relation is obtained:

$\sum_{k=1}^{K} \tilde{g}_{k}\left(\boldsymbol{S}^{\diamond}\right) \leq K \max _{k} \tilde{g}_{k}\left(\boldsymbol{S}^{\diamond}\right)<K \max _{k} \tilde{g}_{k}\left(\boldsymbol{S}^{*}\right)=\sum_{k=1}^{K} \tilde{g}_{k}\left(\boldsymbol{S}^{*}\right)$.

Note that the second inequality and the equality in (39) are due to the assumptions of $\max _{k} \tilde{g}_{k}\left(\boldsymbol{S}^{\diamond}\right)<\max _{k} \tilde{g}_{k}\left(\boldsymbol{S}^{*}\right)$ and $\tilde{g}_{1}\left(\boldsymbol{S}^{*}\right)=\cdots=\tilde{g}_{K}\left(\boldsymbol{S}^{*}\right)$, respectively. Since (39) implies that $\sum_{k=1}^{K} \tilde{g}_{k}\left(\boldsymbol{S}^{\diamond}\right)<\sum_{k=1}^{K} \tilde{g}_{k}\left(\boldsymbol{S}^{*}\right)$, it results in a contradiction due to the definition of $\boldsymbol{S}^{*}$ in (28). Therefore, when $\tilde{g}_{1}\left(\boldsymbol{S}^{*}\right)=$ $\cdots=\tilde{g}_{K}\left(\boldsymbol{S}^{*}\right)$, the relation $\max _{k} \tilde{g}_{k}\left(\boldsymbol{S}^{\diamond}\right)<\max \tilde{g}_{k}\left(\boldsymbol{S}^{*}\right)$ cannot be true. This implies that $\max _{k} \tilde{g}_{k}\left(\boldsymbol{S}^{\diamond}\right)=\max _{k} \tilde{g}_{k}\left(\boldsymbol{S}^{*}\right)$ must be satisfied in this scenario since $\max _{k} \stackrel{k}{\tilde{g}}_{k}\left(\boldsymbol{S}^{\diamond}\right) \leq$ $\max _{k} \tilde{g}_{k}\left(\boldsymbol{S}^{*}\right)$ is always satisfied by definition (as $\boldsymbol{S}^{\diamond}$ is the 
solution of (26)). Then, $\mathrm{P}_{\mathrm{DR}}=\mathrm{P}_{\mathrm{SD}}$ is obtained as follows:

$\mathrm{P}_{\mathrm{SD}}=\max _{k} \tilde{g}_{k}\left(\boldsymbol{S}^{\diamond}\right)=\max _{k} \tilde{g}_{k}\left(\boldsymbol{S}^{*}\right)=\frac{1}{K} \sum_{k=1}^{K} \tilde{g}_{k}\left(\boldsymbol{S}^{*}\right)=\mathrm{P}_{\mathrm{LB}}$

where the third equality is due to $\tilde{g}_{1}\left(\boldsymbol{S}^{*}\right)=\cdots=\tilde{g}_{K}\left(\boldsymbol{S}^{*}\right)$ and the last equality is from (27). Since in general $\mathrm{P}_{\mathrm{LB}} \leq \mathrm{P}_{\mathrm{DR}} \leq$ $\mathrm{P}_{\mathrm{SD}}$ holds (see (27) and Part (i) of Proposition 4), (40) implies that $\mathrm{P}_{\mathrm{DR}}=\mathrm{P}_{\mathrm{SD}}=\mathrm{P}_{\mathrm{LB}}$ when $\tilde{g}_{1}\left(\boldsymbol{S}^{*}\right)=\cdots=\tilde{g}_{K}\left(\boldsymbol{S}^{*}\right)$.

(iv) Assume that $\mathrm{P}_{\mathrm{DR}}=\mathrm{P}_{\mathrm{LB}}$ and $\tilde{g}_{k}\left(\boldsymbol{S}^{\diamond}\right) \neq \tilde{g}_{l}\left(\boldsymbol{S}^{\diamond}\right)$ for some $k, l \in\{1, \ldots, K\}$. Then, the result is derived as follows:

$$
\begin{aligned}
& \mathrm{P}_{\mathrm{SD}}=\max _{k} \tilde{g}_{k}\left(\boldsymbol{S}^{\diamond}\right)>\frac{1}{K} \sum_{k=1}^{K} \tilde{g}_{k}\left(\boldsymbol{S}^{\diamond}\right) \\
& \geq \frac{1}{K} \sum_{k=1}^{K} \tilde{g}_{k}\left(\boldsymbol{S}^{*}\right)=\mathrm{P}_{\mathrm{LB}}=\mathrm{P}_{\mathrm{DR}},
\end{aligned}
$$

where the first inequality is obtained from the assumption that $\tilde{g}_{k}\left(\boldsymbol{S}^{\diamond}\right) \neq \tilde{g}_{l}\left(\boldsymbol{S}^{\diamond}\right)$ for some $k, l \in\{1, \ldots, K\}$, the second inequality and the second equality follow from Proposition 3 , and the final equality is due to the assumption of $\mathrm{P}_{\mathrm{DR}}=\mathrm{P}_{\mathrm{LB}}$.

Proposition 4 quantifies the improvements that can be achieved via the optimal detector randomization approach and states that the worst-case average probability of error can be reduced by a factor of $K$ compared to the optimal single detectors approach that does not perform any detector randomization. Therefore, significant gains can be possible in the presence of a large number of users. In addition, the scenarios in which this maximum improvement ratio can be achieved are specified based on the conditions in Part (ii) of the proposition. It should be noted that the condition of $\tilde{g}_{k}\left(\boldsymbol{S}^{*}\right)=0, \forall k \in\{1 \ldots K\} \backslash\left\{k^{*}\right\}$ and $\tilde{g}_{k^{*}}\left(\boldsymbol{S}^{*}\right)>0$ cannot hold exactly for ML detectors that operate in the presence of Gaussian noise, which has an infinite support. Therefore, the maximum improvement ratio of $K$ may not be achieved exactly in practice; however, it can be quite close to $K$ in certain scenarios (see, e.g., Fig. 3 at 28 dB). Proposition 4 also provides some simple conditions to determine if the optimal detector randomization approach can or cannot provide any improvements over the optimal single detectors approach.

Remark 1: Although the results in Proposition 3 and Proposition 4 are obtained when all the users employ ML detectors, which are specified by the error probability expression $\tilde{g}_{k}$ in (23), the results are also valid for other types of detectors; e.g., the sign detector or the optimal single-threshold detector. In other words, Proposition 3 and Proposition 4 hold for arbitrary $\tilde{g}_{k}$ corresponding to any type of detectors.

In the following proposition, the structure of the optimal detector randomization solution obtained from (20)-(22) is specified in the case of equal crosscorrelations and noise powers.

Proposition 5: Assume that there are at least $K$ detectors at each receiver; that is, $N_{\mathrm{d}} \geq K$. If the crosscorrelations between the pseudo-noise signals for different users are equal; i.e., $\rho_{k, j}=\rho, \forall k \neq j$, and the standard deviations of the noise at the receivers are the same; i.e., $\sigma_{k}=\sigma, \forall k$, then an optimal solution to (20)-(22), which achieves the lower bound in (27), can be expressed as

$$
v_{l}=\frac{1}{K}, \boldsymbol{S}_{l}=\mathrm{CS}_{2 l-2}\left(\boldsymbol{S}^{*}\right) \text { for } l=1, \ldots, K
$$

where $\boldsymbol{S}^{*}$ is as in (28) and $\mathrm{CS}_{2 l-2}\left(\boldsymbol{S}^{*}\right)$ denotes the circular shift of the elements of $\boldsymbol{S}^{*}$ by $2 l-2$ positions. $^{7}$

Proof: When the solution in (42) is employed, the objective function in (20) becomes

$$
\begin{array}{r}
\max _{k \in\{1, \ldots, K\}} \frac{1}{2 K} \int_{-\infty}^{\infty} \sum_{l=1}^{K} \min \left\{p_{0}^{(k)}\left(y \mid \mathrm{CS}_{2 l-2}\left(\boldsymbol{S}^{*}\right)\right),\right. \\
\left.p_{1}^{(k)}\left(y \mid \mathrm{CS}_{2 l-2}\left(\boldsymbol{S}^{*}\right)\right)\right\} d y .
\end{array}
$$

In addition, for equal crosscorrelations and noise variances, $p_{i_{k}}^{(k)}\left(y \mid \boldsymbol{S}_{l}\right)$ in (19) is given by

$$
\begin{aligned}
p_{i_{k}}^{(k)}\left(y \mid \boldsymbol{S}_{l}\right)= & \frac{1}{\sigma \sqrt{2 \pi} 2^{K-1}} \sum_{\mathbf{i}_{k} \in\{0,1\}^{K-1}} \\
& \exp \left\{-\frac{1}{2 \sigma^{2}}\left(y-S_{k, l}^{\left(i_{k}\right)}-\rho \sum_{\substack{j=1 \\
j \neq k}}^{K} S_{j, l}^{\left(i_{j}\right)}\right)^{2}\right\}
\end{aligned}
$$

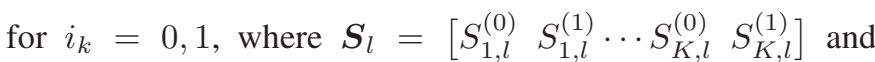
$\mathbf{i}_{k}=\left[\begin{array}{ll}i_{1} \cdots i_{k-1} & i_{k+1} \cdots i_{K}\end{array}\right]$. Then, if $\boldsymbol{S}_{l}=\mathrm{CS}_{2 l-2}\left(\boldsymbol{S}^{*}\right)$ is employed for $l=1, \ldots, K$, where $\boldsymbol{S}^{*} \triangleq$ $\left[S_{1, *}^{(0)} S_{1, *}^{(1)} \cdots S_{K, *}^{(0)} S_{K, *}^{(1)}\right]$, it can be shown based on (44) that the $\sum_{l=1}^{K} \min \left\{p_{0}^{(k)}\left(y \mid \mathrm{CS}_{2 l-2}\left(\boldsymbol{S}^{*}\right)\right), p_{1}^{(k)}\left(y \mid \mathrm{CS}_{2 l-2}\left(\boldsymbol{S}^{*}\right)\right)\right\}$ terms in (43) become equal for $k=1, \ldots, K{ }^{8}$ Therefore, the overall expression in (43) can be stated as

$$
\begin{aligned}
\frac{1}{2 K} \sum_{l=1}^{K} \int_{-\infty}^{\infty} \min \{ & p_{0}^{(k)}\left(y \mid \mathrm{CS}_{2 l-2}\left(\boldsymbol{S}^{*}\right)\right) \\
& \left.p_{1}^{(k)}\left(y \mid \mathrm{CS}_{2 l-2}\left(\boldsymbol{S}^{*}\right)\right)\right\} d y
\end{aligned}
$$

for any $k \in\{1, \ldots, K\}$. From (44), it is easy to verify that (45) is also equal to

$$
\frac{1}{2 K} \sum_{k=1}^{K} \int_{-\infty}^{\infty} \min \left\{p_{0}^{(k)}\left(y \mid \boldsymbol{S}^{*}\right), p_{1}^{(k)}\left(y \mid \boldsymbol{S}^{*}\right)\right\} d y
$$

which can be expressed as $\frac{1}{K} \sum_{k=1}^{K} \tilde{g}_{k}\left(\boldsymbol{S}^{*}\right) \triangleq \mathrm{P}_{\mathrm{LB}}$ based on the definitions in (23) and (27). Hence, it is observed that for the solution in (42), the optimization problem in (20)-(22) achieves the lower bound in Proposition 3; i.e., (42) provides an optimal solution to (20)-(22) that achieves the lower bound in (27), as claimed in the proposition.

Although the optimal solution to the generic problem in (20)-(22) requires a search over a $(2 K+1) K$ dimensional space (assuming $N_{\mathrm{d}} \geq K$ ), a significantly simpler solution can be obtained under the conditions in Proposition 5; namely, the

\footnotetext{
${ }^{7}$ Since $\boldsymbol{S}^{*}$ is feasible; i.e, satisfies $h\left(\boldsymbol{S}^{*}\right) \leq A$ by definition (see (28)), $\mathrm{CS}_{2 l}\left(\boldsymbol{S}^{*}\right)$ 's are feasible as well due to the definition of $h$ in (9).

${ }^{8}$ For example, if $K=2$, then $\mathrm{CS}_{0}\left(\boldsymbol{S}^{*}\right)=$ $\left[\begin{array}{llll}S_{1, *}^{(0)} & S_{1, *}^{(1)} & S_{2, *}^{(0)} & S_{2, *}^{(1)}\end{array}\right]$ and $\mathrm{CS}_{2}\left(\boldsymbol{S}^{*}\right)=\left[\begin{array}{llll}S_{2, *}^{(0)} & S_{2, *}^{(1)} & S_{1, *}^{(0)} & S_{1, *}^{(1)}\end{array}\right]$, for which $\min \left\{p_{0}^{(k)}\left(y \mid \mathrm{CS}_{0}\left(\boldsymbol{S}^{*}\right)\right), p_{1}^{(k)}\left(y \mid \mathrm{CS}_{0}\left(\boldsymbol{S}^{*}\right)\right)\right\}+$ $\min \left\{p_{0}^{(k)}\left(y \mid \mathrm{CS}_{2}\left(\boldsymbol{S}^{*}\right)\right), p_{1}^{(k)}\left(y \mid \mathrm{CS}_{2}\left(\boldsymbol{S}^{*}\right)\right)\right\}$ is the same for $k=1$
} and $k=2$, as can be observed from (44). 
following algorithm can be employed: (i) Calculate $S^{*}$ from (28). (ii) Obtain the optimal solution as in (42). ${ }^{9}$ It is noted that this algorithm requires a search over a $2 K$ dimensional space in order to calculate $S^{*}$. In addition, if symmetric signaling is employed, the search space dimensions reduce to $(K+1) K$ and $K$ for the problems in (20)-(22) and in (28), respectively.

Remark 2: Under the conditions in Proposition 5, if $S^{*}$ is a solution of (28), any permutation of the signal amplitude pairs for different users is a solution as well. ${ }^{10}$ For example, if $\boldsymbol{S}^{*}=\left[S_{1, *}^{(0)} S_{1, *}^{(1)} S_{2, *}^{(0)} S_{2, *}^{(1)} S_{3, *}^{(0)} S_{3, *}^{(1)}\right]=\left[\begin{array}{lllll}-1 & 1 & -2 & 2 & -3\end{array}\right]$,

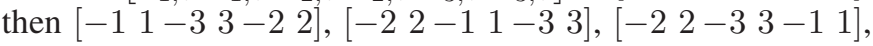

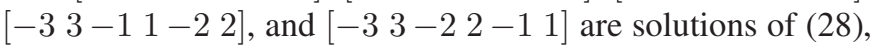
too.

The following proposition presents necessary and sufficient conditions for the uniqueness of the solution in (42).

Proposition 6: Consider scenarios in which performance improvements are achieved via optimal detector randomization over the optimal single detectors approach. Under the conditions in Proposition 5, the optimal solution in (42) is unique if and only if

- the solution of (28), $\boldsymbol{S}^{*}$, is unique up to permutations of signal amplitude pairs (see Remark 2), and

- the signal amplitude pairs in $\boldsymbol{S}^{*}$ are the same except for one of them. ${ }^{11}$

Proof: Please see Appendix A.

Proposition 6 guarantees the uniqueness of the optimal solution in (42) based on the uniqueness of the solution $S^{*}$ of (28) and the structure of this optimal solution. As an example, for $K=4$, if $S^{*}=\left[\begin{array}{llllll}-1 & 1-1 & 1-1 & 1-2 & 2\end{array}\right]$ is the unique solution of (28) up to permutations of signal amplitude pairs (i.e., the only solutions of (28) are $\left[\begin{array}{llllllll}-1 & 1 & -1 & 1 & -1 & 1 & -2 & 2\end{array}\right]$, $\left[\begin{array}{lllll}-1 & 1-1 & 1-2 & 2-1 & 1\end{array}\right],\left[\begin{array}{llllll}-1 & 1-2 & 2-1 & 1-1 & 1\end{array}\right]$, and

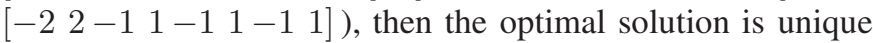
as a result of Proposition 6 since the signal amplitude pairs in $S^{*}$ are the same except for one of them. Also, from Proposition 5, the optimal solution in (42) is given by $v_{1}=$ $v_{2}=v_{3}=v_{4}=0.25, \boldsymbol{S}_{1}=\left[\begin{array}{lllllll}-1 & 1 & -1 & 1 & -1 & 1 & -2\end{array}\right]$

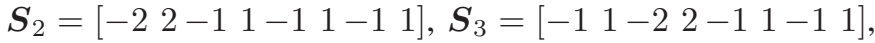

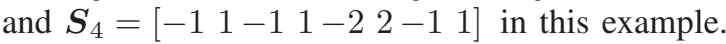

\section{Performance Evaluation}

In this section, numerical results are presented to investigate the theoretical results obtained in the previous sections and to compare the proposed optimal detector randomization approach against other approaches that do not perform any detector randomization. Specifically, the following approaches are considered in the simulations.

Optimal Detector Randomization: This scheme refers to the proposed optimization problem in (13)-(15), which can be

\footnotetext{
${ }^{9}$ The definition of the circular shift in Proposition 5 can be a right circular shift or a left circular shift without affecting the optimality of the solution in (42).

${ }^{10}$ This is implied by the proof of Proposition 5 based on the equivalence of (45) and (46) (see (23) and (28) as well).

${ }^{11}$ The case in which $\boldsymbol{S}^{*}$ is unique and the signal amplitude pairs in $\boldsymbol{S}^{*}$ are all the same is not considered since no improvement is achieved via detector randomization in that scenario (i.e., the condition in Part (iii) of Proposition 4 is satisfied). Specifically, $\boldsymbol{S}^{*}$ is employed all the time and each user runs a single ML detector corresponding to $\boldsymbol{S}^{*}$.
}

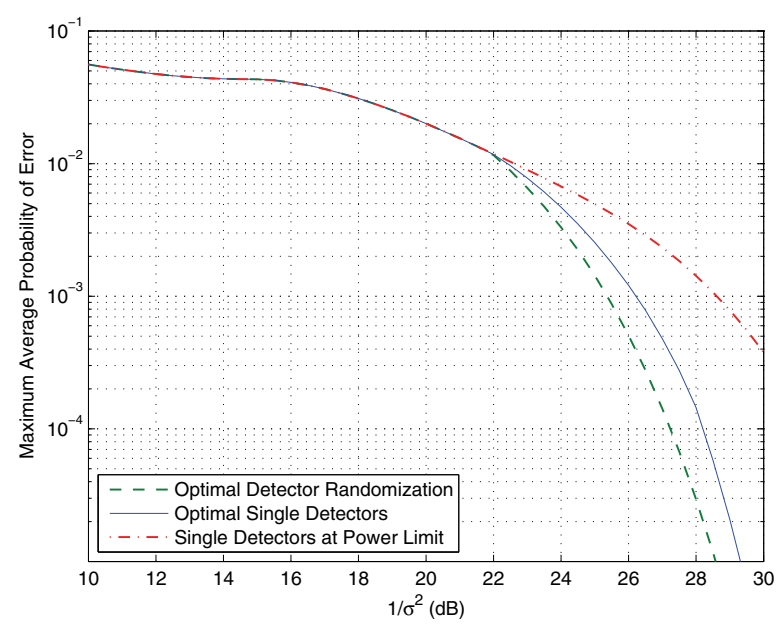

Fig. 3. Maximum average probability of error versus $1 / \sigma^{2}$ for the optimal detector randomization, optimal single detectors, and single detectors at power limit approaches, where $K=5, \rho_{k, j}=0.27$ for all $k \neq j$, and $A=5$.

solved via (20)-(22), as stated in Proposition 2. It is noted that when the conditions in Proposition 5 are satisfied, the optimal solution can also be obtained via (42), which has significantly lower computational complexity.

Optimal Single Detectors: In this approach, a single detector is employed by each user; hence, no detector randomization is performed. The solution is obtained from (25) (equivalently, (26)). Namely, the optimal signals and the corresponding single detectors (ML rules) are calculated in this approach.

Single Detectors at Power Limit: This approach employs a single detector for each user, and equalizes the signalto-interference-plus-noise ratios (SINRs) at all the detectors. In addition, all the available power is utilized. Specifically, in this scheme, the signal amplitudes are chosen in such a way that $\operatorname{SINR}_{1}=\cdots=\operatorname{SINR}_{K}$ and $h(\boldsymbol{S})=A$, where $\mathrm{SINR}_{k}$ is the SINR for user $k$ and $h(\boldsymbol{S})$ is as in (9). The SINR for user $k$ can be calculated from (3) as $\operatorname{SINR}_{k}=$ $\mathrm{E}\left\{\left|S_{k}^{\left(i_{k}\right)}\right|^{2}\right\} /\left(\mathrm{E}\left\{\left|\sum_{j \neq k} \rho_{k, j} S_{j}^{\left(i_{j}\right)}\right|^{2}\right\}+\sigma_{k}^{2}\right)$ for $k=1, \ldots, K$, which becomes $\operatorname{SINR}_{k}=\left|S_{k}^{(1)}\right|^{2} /\left(\sum_{j \neq k} \rho_{k, j}^{2}\left|S_{j}^{(1)}\right|^{2}+\sigma_{k}^{2}\right)$ for symmetric signaling. In general, the single detectors at power limit approach has low computational complexity compared to the other approaches; however, it can result in degraded performance as investigated in the following.

In the simulations, symmetric signaling with equiprobable information symbols is considered for all users, and the standard deviations of the noise at the receivers are set to the same value; i.e., $\sigma_{k}=\sigma, k=1, \ldots, K$. In addition, as stated after (3), $\rho_{k, j}$ 's are taken as one for $k=j$; that is, $\rho_{k, k}=1$ for $k=1, \ldots, K$.

First, a 5-user scenario is considered (that is, $K=5$ ), and the crosscorrelations between the pseudo-noise signals for different users are set to 0.27 ; i.e., $\rho_{k, j}=0.27$ for $k \neq j$. Also, the average power constraint $A$ in (6) is taken as 5. In Fig. 3, the maximum average probability of error is plotted versus $1 / \sigma^{2}$ for the optimal detector randomization, optimal single detectors, and single detectors at power limit approaches. From the figure, it is observed that the optimal detector randomization approach achieves the best performance among all the approaches, and the optimal single detectors approach 
TABLE I

SOLUTION OF THE OPTIMAL SINGLE DETECTORS APPROACH IN (26) FOR THE SCENARIO IN FIG. 3. (ONLY THE SIGNAL AMPLITUDES FOR BIT 1 OF THE USERS ARE SHOWN DUE TO SYMMETRY.)

\begin{tabular}{|l|c|c|c|c|c|}
\hline $1 / \sigma^{2}(\mathrm{~dB})$ & $S_{1, \diamond}^{(1)}$ & $S_{2, \diamond}^{(1)}$ & $S_{3, \diamond}^{(1)}$ & $S_{4, \diamond}^{(1)}$ & $S_{5, \diamond}^{(1)}$ \\
\hline 18 & 1 & 1 & 1 & 1 & 1 \\
20 & 1 & 1 & 1 & 1 & 1 \\
22 & 1.1167 & 0.9686 & 0.9686 & 0.9686 & 0.9686 \\
24 & 1.1321 & 0.9642 & 0.9642 & 0.9642 & 0.9642 \\
26 & 1.1421 & 0.9612 & 0.9612 & 0.9612 & 0.9612 \\
28 & 0.1514 & 1.1154 & 1.1154 & 1.1154 & 1.1154 \\
\hline
\end{tabular}

TABLE II

SOLUTION OF (28), $\boldsymbol{S}^{*}$, FOR THE SCENARIO IN FIG. 3. (ONLY THE SIGNAL AMPLITUDES FOR BIT 1 OF THE USERS ARE SHOWN DUE TO SYMMETRY.) NOTE THAT $\boldsymbol{S}^{*}$ SPECIFIES THE SOLUTION OF THE OPTIMAL DETECTOR RANDOMIZATION APPROACH AS IN (42).

\begin{tabular}{|l|c|c|c|c|c|}
\hline $1 / \sigma^{2}(\mathrm{~dB})$ & $S_{1, *}^{(1)}$ & $S_{2, *}^{(1)}$ & $S_{3, *}^{(1)}$ & $S_{4, *}^{(1)}$ & $S_{5, *}^{(1)}$ \\
\hline 18 & 1 & 1 & 1 & 1 & 1 \\
20 & 1 & 1 & 1 & 1 & 1 \\
22 & 0.1531 & 1.1154 & 1.1154 & 1.1154 & 1.1154 \\
24 & 0.1522 & 1.1154 & 1.1154 & 1.1154 & 1.1154 \\
26 & 0.1516 & 1.1155 & 1.1155 & 1.1155 & 1.1155 \\
28 & 0.1513 & 1.1155 & 1.1155 & 1.1155 & 1.1155 \\
\hline
\end{tabular}

outperforms the single detectors at power limit approach for small noise variances. In addition, the calculations show that for high noise variances the nonimprovability condition in Part (iii) of Proposition 4 is satisfied, while for small noise variances the improvability condition stated in Part (iv) of the same proposition is valid. It is also noted that the improvement ratio, which is the ratio between the maximum error probabilities of the optimal single detectors and optimal detector randomization approaches, satisfies the inequality (36) in Proposition 4. In particular, the maximum improvement ratio of 5 is approximately achieved at $1 / \sigma^{2}=28 \mathrm{~dB}$.

In order to investigate the results in Fig. 3 in more detail, Table I presents the solution $S^{\diamond}$ of the optimal single detectors approach in (26) for various noise variances, where $\boldsymbol{S}^{\diamond}=\left[S_{1, \diamond}^{(0)} S_{1, \diamond}^{(1)} \cdots S_{K, \diamond}^{(0)} S_{K, \diamond}^{(1)}\right]$. Since symmetric signaling is employed, only the signal amplitudes corresponding to bit 1 of the users are shown in the table. (The signal amplitudes for bit 0 are given by $S_{k, \diamond}^{(0)}=-S_{k, \diamond}^{(1)}$ for $k=1,2,3,4,5$.). In addition, Table II illustrates the solution of (28), $\boldsymbol{S}^{*}$, which specifies the solution of the optimal detector randomization approach as described in (42) in Proposition 5. Again only the signal amplitudes corresponding to bit 1 of the users are shown due to symmetry. From Tables I and II, it is observed that both the optimal single detectors and the optimal detector randomization approaches converge to the single detectors at power limit approach for high noise variances. This is due to the fact that the Gaussian noise becomes dominant as the noise variance increases and the multiuser interference plus noise term becomes approximately a Gaussian random variable, in which case the optimal solution is to assign equal powers for all users at the maximum power limit. Also, it is noted that the nonimprovability condition in Part (iii) of Proposition 4 is satisfied for that scenario. On the other hand, for small noise variances, the solutions become different from that of the single detectors at power limit approach, and improvements are achieved as observed in Fig. 3. In addition, Table II implies that the conditions in

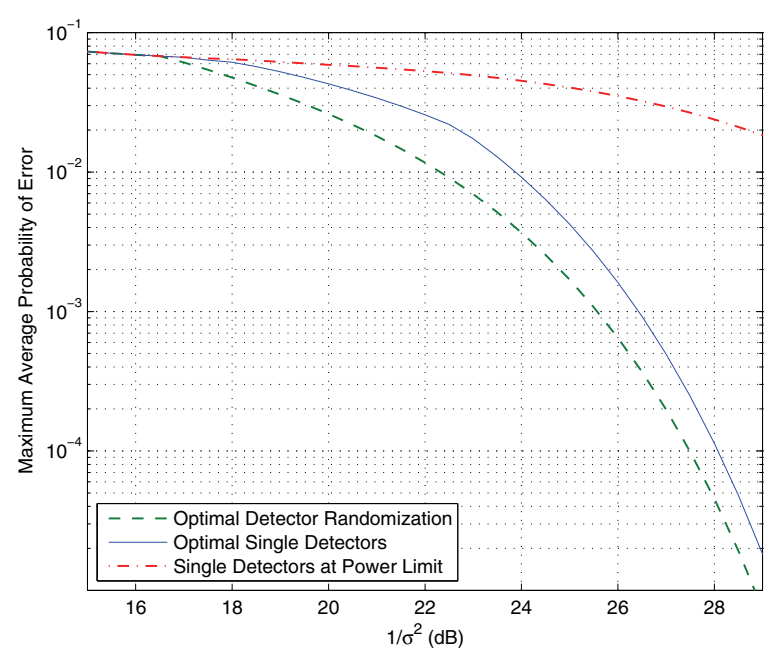

Fig. 4. Maximum average probability of error versus $1 / \sigma^{2}$ for the optimal detector randomization, optimal single detectors, and single detectors at power limit approaches, where $K=5, \rho_{k, j}=0.35$ for all $k \neq j$, and $A=5$.

Proposition 6 are satisfied for small noise variances; hence, the solution of the optimal detector randomization approach specified in (28) is unique in those scenarios. For example, at $1 / \sigma^{2}=24 \mathrm{~dB}$, the unique solution of the optimal detector randomization approach is specified by $v_{l}=0.2$ and $\boldsymbol{S}_{l}=$ $\mathrm{CS}_{2 l-2}([-0.15220 .1522-1.11541 .1154-1.11541 .1154-$ $1.11541 .1154-1.11541 .1154])$ for $l=1,2,3,4,5$. Another important observation can be made from Table II regarding the signal values for the optimal detector randomization approach. When the noise variance is smaller than a certain value, the optimal solution does not vary significantly with the noise level. Hence, perfect knowledge of the noise level may not be required for achieving a near optimal performance. Finally, it is observed from Tables I and II that the optimal signal values are the same for many (or, all) of the users at a given noise variance, which is mainly due to the structures of the optimization problems in (26) and (28), and the facts that the crosscorrelations between the pseudo-noise signals for different users are equal, and the standard deviations of the noise at the receivers are the same. In other words, the optimization problems in (26) and (28) tend to yield equalizer rules (for all or some of the users) in the considered scenario.

Next, another scenario with $K=5$ users is considered, where $\rho_{k, j}=0.35$ for $k \neq j$, and $A=5$. In Fig. 4, the maximum average probability of error is illustrated for the optimal detector randomization, optimal single detectors, and single detectors at power limit approaches. Similar observations to those for Fig. 3 can be made. The main difference is that improvements are achieved for a larger range of noise variances in this scenario. In addition, the solutions of the optimal single detectors and the optimal detector randomization approaches are specified in Tables III and IV for the scenario in Fig. 4 for some values of $1 / \sigma^{2}$. Again similar observations to those in the previous scenario can be made. However, in this case, the solution in (28) is not unique since the second uniqueness condition in Proposition 6 is not satisfied, as observed from Table IV.

Then, a scenario with $K=6$ users is considered, where $\rho_{k, j}=0.21$ for $k \neq j$, and $A=6$. In Fig. 5, the maximum 
TABLE III

SOLUTION OF THE OPTIMAL SINGLE DETECTORS APPROACH IN (26) FOR THE SCENARIO IN FIG. 4.

\begin{tabular}{|l|c|c|c|c|c|}
\hline $1 / \sigma^{2}(\mathrm{~dB})$ & $S_{1, \diamond}^{(1)}$ & $S_{2, \diamond}^{(1)}$ & $S_{3, \diamond}^{(1)}$ & $S_{4, \diamond}^{(1)}$ & $S_{5, \diamond}^{(1)}$ \\
\hline 15 & 1 & 1 & 1 & 1 & 1 \\
20 & 1.1099 & 1.1099 & 0.9195 & 0.9195 & 0.9195 \\
25 & 0.2180 & 0.2180 & 1.2787 & 1.2787 & 1.2787 \\
30 & 0.2218 & 0.2218 & 1.2782 & 1.2782 & 1.2782 \\
\hline
\end{tabular}

TABLE IV

Solution of (28), $\boldsymbol{S}^{*}$, FOR the SCEnARIo in Fig. 4. Note that $\boldsymbol{S}^{*}$ SPECIFIES THE SOLUTION OF THE OPTIMAL DETECTOR RANDOMIZATION APPROACH AS IN (42).

\begin{tabular}{|l|c|c|c|c|c|}
\hline $1 / \sigma^{2}(\mathrm{~dB})$ & $S_{1, *}^{(1)}$ & $S_{2, *}^{(1)}$ & $S_{3, *}^{(1)}$ & $S_{4, *}^{(1)}$ & $S_{5, *}^{(1)}$ \\
\hline 15 & 1 & 1 & 1 & 1 & 1 \\
20 & 0.2084 & 0.2084 & 1.2797 & 1.2797 & 1.2797 \\
25 & 0.2180 & 0.2180 & 1.2787 & 1.2787 & 1.2787 \\
30 & 0.2218 & 0.2218 & 1.2782 & 1.2782 & 1.2782 \\
\hline
\end{tabular}

average probability of error is illustrated for the optimal detector randomization, optimal single detectors, and single detectors at power limit approaches. Similar observations as in the previous scenarios are made. The main difference in this scenario is that the improvement ratio is smaller than those in Fig. 3 and Fig. 4. Also, the solutions of the optimal single detectors and the optimal detector randomization approaches are specified in Tables V and VI for the scenario in Fig. 5 for some values of $1 / \sigma^{2}$.

Finally, a system with 10 users is considered, where $\rho_{k, j}=$ 0.17 for $k \neq j$, and $A=10$. In Fig. 6, the maximum average probability of error is plotted versus $1 / \sigma^{2}$ for the optimal detector randomization, optimal single detectors, and single detectors at power limit approaches. Again similar observations to those for the previous scenarios are made. The main difference in this case is that the error rates are higher for a given noise level than those in the previous scenarios, which is mainly due to the large number of users.

\section{CONCLUSIONS AND EXTENSIONS}

Optimal detector randomization has been studied for the downlink of a DSSS system. An optimization problem has been formulated in order to obtain the optimal signal amplitudes, detectors, and detector randomization factors. Since this joint optimization problem is quite challenging in general, a simplified problem has been proposed, in which the search is performed over signal amplitudes and detector randomization factors only, and then the ML detectors corresponding to the optimal signal amplitudes are employed at the receivers. It has been shown that this simplified approach provides the optimal solution to the generic problem when detector randomization is performed over at most $\min \left\{K, N_{\mathrm{d}}\right\}$ detector sets, where $K$ is the number of users and $N_{\mathrm{d}}$ is the number of detectors at each receiver. Then, the performance of the optimal detector randomization approach has been investigated, and a lower bound has been obtained for the minimum worstcase average probability of error. Also, it has been shown that the optimal detector randomization approach can improve the performance of the optimal single detectors approach by up to $K$ times. In addition, various sufficient conditions have been obtained for the improvability and nonimprovability via detector randomization. Furthermore, in the special case of equal

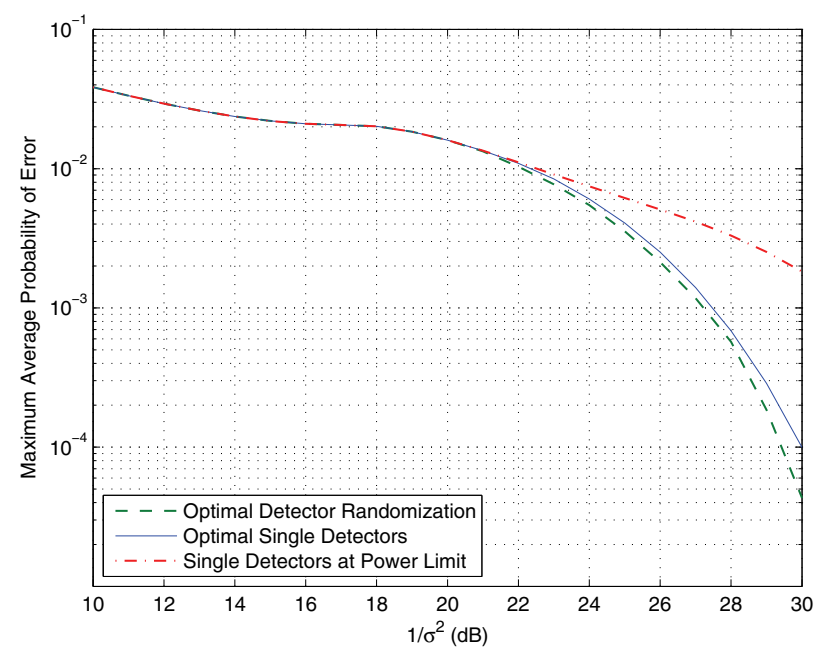

Fig. 5. Maximum average probability of error versus $1 / \sigma^{2}$ for the optimal detector randomization, optimal single detectors, and single detectors at power limit approaches, where $K=6, \rho_{k, j}=0.21$ for all $k \neq j$, and $A=6$.

TABLE V

SOLUTION OF THE OPTIMAL SINGLE DETECTORS APPROACH IN (26) FOR THE SCENARIO IN FIG. 5.

\begin{tabular}{|l|c|c|c|c|c|c|}
\hline $1 / \sigma^{2}(\mathrm{~dB})$ & $S_{1, \diamond}^{(1)}$ & $S_{2, \diamond}^{(1)}$ & $S_{3, \diamond}^{(1)}$ & $S_{4, \diamond}^{(1)}$ & $S_{5, \diamond}^{(1)}$ & $S_{6, \diamond}^{(1)}$ \\
\hline 18 & 1 & 1 & 1 & 1 & 1 & 1 \\
20 & 1 & 1 & 1 & 1 & 1 & 1 \\
22 & 1.0662 & 0.9862 & 0.9862 & 0.9862 & 0.9862 & 0.9862 \\
24 & 1.0978 & 0.9793 & 0.9793 & 0.9793 & 0.9793 & 0.9793 \\
26 & 1.1353 & 0.9707 & 0.9707 & 0.9707 & 0.9707 & 0.9707 \\
28 & 1.1602 & 0.9648 & 0.9648 & 0.9648 & 0.9648 & 0.9648 \\
\hline
\end{tabular}

crosscorrelations and noise powers, a simple solution has been provided for the optimal detector randomization problem, and necessary and sufficient conditions have been presented for the uniqueness of that solution. Finally, numerical examples have been presented in order to illustrate the improvements achieved via detector randomization.

Although the downlink of a DSSS system is considered in this study, the results can also be applied to the uplink of a synchronous DSSS under certain assumptions. Specifically, suppose that the receiver (the base station or the access point) employs a bank of $K$ correlators corresponding to the pseudonoise signals of the users and then performs the bit decision for user $k$ based on the $k^{\text {th }}$ correlator output via detector randomization among $N_{\mathrm{d}}$ detectors, where $k \in\{1, \ldots, K\}$. In this scenario, the theoretical results in Section III and Section IV can be extended to the uplink as well. However, when an asynchronous system is considered or when the receiver employs multiuser detection approaches [33], the results in this study cannot be directly applied. Therefore, optimal detector randomization in such scenarios is considered as a future work.

The results in this study can also be extended to cover scenarios in which each user performs $M$-ary modulation for $M>2$. In that case, the definitions of $\phi_{l}^{(k)}$ in (4), $\boldsymbol{S}_{l}$ at the beginning of Section III, $h$ in (9), $g_{k, l}$ in (11), and $\tilde{g}_{k}$ in (23) can be updated accordingly, and the theoretical results in the previous sections can still be employed based on these new definitions. 
TABLE VI

Solution of (28), $\boldsymbol{S}^{*}$, FOR the SCEnARIO In Fig. 5. Note that $\boldsymbol{S}^{*}$ SPECIFIES THE SOLUTION OF THE OPTIMAL DETECTOR RANDOMIZATION APPROACH AS IN (42).

\begin{tabular}{|c|c|c|c|c|c|c|}
\hline $1 / \sigma^{2}(\mathrm{~dB})$ & $S_{1, *}^{(1)}$ & $S_{2, *}^{(1)}$ & $S_{3, *}^{(1)}$ & $S_{4, *}^{(1)}$ & $S_{5, *}^{(1)}$ & $S_{6, *}^{(1)}$ \\
\hline 18 & 1 & 1 & 1 & 1 & 1 & 1 \\
20 & 1 & 1 & 1 & 1 & 1 & 1 \\
22 & 1.1117 & 0.9761 & 0.9761 & 0.9761 & 0.9761 & 0.9761 \\
24 & 1.1283 & 0.9723 & 0.9723 & 0.9723 & 0.9723 & 0.9723 \\
26 & 1.1430 & 0.9689 & 0.9689 & 0.9689 & 0.9689 & 0.9689 \\
28 & 1.1606 & 0.9647 & 0.9647 & 0.9647 & 0.9647 & 0.9647 \\
\hline
\end{tabular}

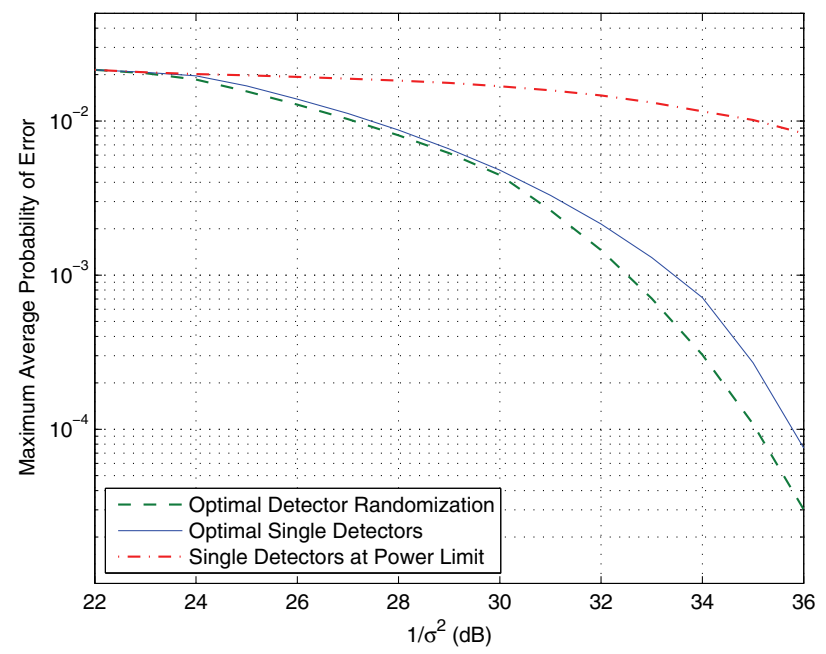

Fig. 6. Maximum average probability of error versus $1 / \sigma^{2}$ for the optimal detector randomization, optimal single detectors, and single detectors at power limit approaches, where $K=10, \rho_{k, j}=0.17$ for all $k \neq j$, and $A=10$.

\section{APPENDIX}

\section{A. Proof of Proposition 6}

First, it is shown that the optimal solution in (42) is unique if the conditions in the proposition are satisfied. To that aim, define the following sets

$$
\begin{aligned}
& \mathcal{S}_{\text {equ }}=\left\{\boldsymbol{S} \in \mathcal{S}_{A}: \sum_{k=1}^{K} \tilde{g}_{k}(\boldsymbol{S})=\sum_{k=1}^{K} \tilde{g}_{k}\left(\boldsymbol{S}^{*}\right)\right\} \\
& \mathcal{S}_{\text {lar }}=\left\{\boldsymbol{S} \in \mathcal{S}_{A}: \sum_{k=1}^{K} \tilde{g}_{k}(\boldsymbol{S})>\sum_{k=1}^{K} \tilde{g}_{k}\left(\boldsymbol{S}^{*}\right)\right\}
\end{aligned}
$$

where $\tilde{g}_{k}$ is given by (23) and $\mathcal{S}_{A}$ is as defined in Proposition 3. Note that each $S \in \mathcal{S}_{A}$ must belong to either $\mathcal{S}_{\text {equ }}$ or $\mathcal{S}_{\text {lar }}$ due to the definition of $\boldsymbol{S}^{*}$ in (28). Let $\left\{v_{l}, \boldsymbol{S}_{l}\right\}_{l=1}^{K}$ denote the optimal solution of (20)-(22). Then, it is proved that $\boldsymbol{S}_{l} \in \mathcal{S}_{\text {equ }}$ must hold for all $l \in\{1, \ldots, K\}$ since it would otherwise lead to a scenario in which the optimal solution of (20)-(22), $P_{D R}$, could not achieve the lower bound in (27) as shown below:

$$
\begin{aligned}
\mathrm{P}_{\mathrm{DR}}=\max _{k \in\{1, \ldots, K\}} & \sum_{l=1}^{K} v_{l} \tilde{g}_{k}\left(\boldsymbol{S}_{l}\right) \geq \frac{1}{K} \sum_{l=1}^{K} v_{l} \sum_{k=1}^{K} \tilde{g}_{k}\left(\boldsymbol{S}_{l}\right) \\
& >\frac{1}{K} \sum_{l=1}^{K} v_{l} \sum_{k=1}^{K} \tilde{g}_{k}\left(\boldsymbol{S}^{*}\right)=\mathrm{P}_{\mathrm{LB}}
\end{aligned}
$$

Here, the strict inequality is obtained under the assumption that there exists $l \in\{1, \ldots, K\}$ such that $\boldsymbol{S}_{l} \notin \mathcal{S}_{\text {equ }}$ (i.e.,
$\boldsymbol{S}_{l} \in \mathcal{S}_{\text {lar }}$ ). However, as stated in Proposition 5, the lower bound must be achieved in the considered scenario. Therefore, (49) presents a contradiction, implying that $\boldsymbol{S}_{l} \in \mathcal{S}_{\text {equ }}$ must hold for all $l \in\{1, \ldots, K\}$.

Next, define set $\mathcal{S}_{\text {per }}$ as follows: $\mathcal{S}_{\text {per }}=\left\{\boldsymbol{S} \in \mathcal{S}_{A}\right.$ : $S$ is a permutation of signal amplitude pairs in $\left.S^{*}\right\}$. From Remark 2, it is noted that the elements of $\mathcal{S}_{\text {per }}$ correspond to all possible $\boldsymbol{S} \in \mathcal{S}_{A}$ that satisfy $\sum_{k=1}^{K} \tilde{g}_{k}(\boldsymbol{S})=$ $\sum_{k=1}^{K} \tilde{g}_{k}\left(\boldsymbol{S}^{*}\right)$; hence, $\mathcal{S}_{\text {per }}=\mathcal{S}_{\text {equ }}$. Then, based on the argument in the previous paragraph, it is concluded that the optimal solution of (20)-(22), $\left\{v_{l}, \boldsymbol{S}_{l}\right\}_{l=1}^{K}$, must satisfy $S_{l} \in \mathcal{S}_{\text {per }}$ for all $l \in\{1, \ldots, K\}$. If the conditions in the proposition are satisfied (i.e., $\boldsymbol{S}^{*}$ is unique up to permutations of the signal amplitude pairs, which are the same except for one of them), there exist exactly $K$ elements in $\mathcal{S}_{\text {per }}$, which correspond to the circular shifts of $\boldsymbol{S}^{*}$ by $2 l-2$ elements for $l=1, \ldots, K$; that is, $\mathcal{S}_{\text {per }}=\left\{\mathrm{CS}_{2 l-2}\left(\boldsymbol{S}^{*}\right), l=1, \ldots, K\right\}$. In order to specify the randomization factors, $v_{1}, \ldots, v_{K}$, of the optimal solution in this scenario, define $\mathbf{v}$ as $\mathbf{v}=\left[v_{1} \cdots v_{K}\right]^{T}$ and $\mathbf{G}$ as a $K \times K$ matrix with its element in row $k$ and column $l$ being equal to $\tilde{g}_{k}\left(\mathrm{CS}_{2 l-2}\left(\boldsymbol{S}^{*}\right)\right){ }^{12}$ Then, based on Proposition 3, the optimal weights must satisfy

$$
\mathbf{G} \mathbf{v}=\mathbf{p}_{\mathrm{LB}} \text { and } \mathbf{1}^{T} \mathbf{v}=1,
$$

where $\mathbf{1} \triangleq[1 \cdots 1]^{T}$ and $\mathbf{p}_{\mathrm{LB}} \triangleq\left[\mathrm{P}_{\mathrm{LB}} \cdots \mathrm{P}_{\mathrm{LB}}\right]^{T}$ with $\mathrm{P}_{\mathrm{LB}}=\frac{1}{K} \sum_{k=1}^{K} \tilde{g}_{k}\left(\boldsymbol{S}^{*}\right)$ as in (27). Note that each element of Gv corresponds to the average error probability of a user, which should be equal to $\mathrm{P}_{\mathrm{LB}}$, since the lower bound in (27) is achieved, i.e., $\mathrm{P}_{\mathrm{DR}}=\mathrm{P}_{\mathrm{LB}}$, in this scenario (see the achievability condition in Proposition 3). It can be shown that $\mathbf{G}$ is a circulant matrix [41] based on the following lemma:

Lemma 1: Under the conditions in Proposition 5, $\tilde{g}_{k}\left(\mathrm{CS}_{2 l-2}\left(\boldsymbol{S}^{*}\right)\right)=\tilde{g}_{j}\left(\mathrm{CS}_{2 m-2}\left(\boldsymbol{S}^{*}\right)\right)$ if $(l-k)_{\bmod K}=$ $(m-j)_{\bmod K}$ for $k, l, j, m \in\{1, \ldots, K\}$.

Proof: From (23), $\tilde{g}_{k}\left(\mathrm{CS}_{2 l-2}\left(\boldsymbol{S}^{*}\right)\right)$ can be expressed as

$\tilde{g}_{k}\left(\mathrm{CS}_{2 l-2}\left(\boldsymbol{S}^{*}\right)\right)=$

$0.5 \int_{-\infty}^{\infty} \min \left\{p_{0}^{(k)}\left(y \mid \mathrm{CS}_{2 l-2}\left(\boldsymbol{S}^{*}\right)\right), p_{1}^{(k)}\left(y \mid \mathrm{CS}_{2 l-2}\left(\boldsymbol{S}^{*}\right)\right)\right\} d y$

where $p_{i_{k}}^{(k)}$ is given by (44) under the conditions in Proposition 5. From (44) and (51), it is observed that $\tilde{g}_{k}\left(\mathrm{CS}_{2 l-2}\left(\boldsymbol{S}^{*}\right)\right)$ and $\tilde{g}_{j}\left(\mathrm{CS}_{2 m-2}\left(\boldsymbol{S}^{*}\right)\right)$ are equal if the $k^{\text {th }}$ signal amplitude pair in $\mathrm{CS}_{2 l-2}\left(\boldsymbol{S}^{*}\right)$ is the same as the $j^{\text {th }}$ signal amplitude pair in $\mathrm{CS}_{2 m-2}\left(\boldsymbol{S}^{*}\right)$. Since the $k^{\text {th }}$ and the $j^{\text {th }}$ signal amplitude pairs in $\mathrm{CS}_{2 l-2}\left(\boldsymbol{S}^{*}\right)$ and $\mathrm{CS}_{2 m-2}\left(\boldsymbol{S}^{*}\right)$, respectively, become the same for $(l-k) \bmod K=(m-j) \bmod K$ due to the nature of the circular shift operator, $\tilde{g}_{k}\left(\mathrm{CS}_{2 l-2}\left(\boldsymbol{S}^{*}\right)\right)=\tilde{g}_{j}\left(\mathrm{CS}_{2 m-2}\left(\boldsymbol{S}^{*}\right)\right)$ is obtained for $(l-k) \bmod K=(m-j) \bmod K$, where $k, l, j, m \in$ $\{1, \ldots, K\}$.

In addition to being a circulant matrix, $G$ also has the property that its elements in each row are either all the same or the same except for one of them under the second condition in the proposition (i.e., when the signal amplitude pairs in $S^{*}$ are the same except for one of them). This observation follows directly from (23) and (44). Therefore, one of the rows of $\mathbf{G}$, say the first one, is in the form of $[a b \cdots b]$, and

\footnotetext{
${ }^{12}$ Note that the elements of $\mathbf{G}$ are strictly positive based on (23) and (44).
} 
the other rows are the circular shifts of this row in such a way that $\mathbf{G}$ is a circulant matrix. First consider the case in which $a \neq b$. Then, it is concluded that $\mathbf{G}$ is nonsingular since its eigenvalues are all nonzero. (In particular, one eigenvalue is $a+(K-1) b$ and the remaining ones are $a-b$.) Hence, there exists a unique solution of (50). Based on the fact that $\frac{1}{K} \sum_{l=1}^{K} \tilde{g}_{k}\left(\operatorname{CS}_{2 l-2}\left(\boldsymbol{S}^{*}\right)\right)=\frac{1}{K} \sum_{j=1}^{K} \tilde{g}_{j}\left(\boldsymbol{S}^{*}\right)$ for each $k \in\{1, \ldots, K\}$ (which can be verified from (23) and (44)), the unique solution of (50) is obtained as $\mathbf{v}=\left[\frac{1}{K} \cdots \frac{1}{K}\right]^{T}$. For $a=b$, all the elements of $\mathbf{G}$; hence, all the $\tilde{g}_{k}\left(\mathrm{CS}_{2 l-2}\left(\boldsymbol{S}^{*}\right)\right)$ terms, are the same. Therefore, no improvement is achieved via detector randomization in that scenario, and the optimal solution can be achieved by employing $S^{*}$ all the time. Hence, this trivial scenario is excluded as stated at the beginning of Proposition 6; that is, $a=b$ does not hold for scenarios considered in the proposition.

In order to prove the necessity of the conditions in the proposition, first assume that $S^{*}$ is not unique up to permutations of signal amplitude pairs. Then, a different solution can be obtained for each distinct $S^{*}$ as described above. Namely, a distinct solution is calculated as in (42) for each $S^{*}$. Therefore, the solution is not unique if the first condition in Proposition 6 is not satisfied. Next, assume that $S^{*}$ is unique up to permutations of signal amplitude pairs but it does not satisfy the second condition in the proposition; that is, there are at least three distinct signal amplitude pairs in $S^{*}$ or two distinct signal amplitude pairs each with multiple repetitions. Then, the permutations of the signal amplitude pairs in $S^{*}$ result in more than $K$ different signal vectors; i.e., there exist more than $K$ elements in set $\mathcal{S}_{\text {per }}$, which is as defined above. (In particular, if there exist $N_{\mathrm{p}}$ distinct signal amplitude pairs in $S^{*}$, each of which has $R_{1}, \ldots, R_{N_{\mathrm{p}}}$ repetitions, respectively, then there are $K ! /\left(R_{1} ! \cdots R_{N_{\mathrm{p}}}\right.$ !) different permutations of signal amplitude pairs; i.e., $\left|\mathcal{S}_{\text {per }}\right|=K ! /\left(R_{1} ! \cdots R_{N_{\mathrm{p}}}\right.$ !). $)$ In this case, there exist at least two distinct signal vectors $S_{x_{1}}^{*}$ and $S_{x_{2}}^{*}$, which are not circular shifts of each other. Then, the circular shifts of $S_{x_{1}}^{*}$ and $S_{x_{2}}^{*}$ can be employed in order to obtain two distinct solutions based on (42). Hence, it is concluded that the solution is not unique if the second condition in the proposition is not satisfied.

\section{REFERENCES}

[1] M. Azizoglu, "Convexity properties in binary detection problems," IEEE Trans. Inf. Theory, vol. 42, no. 4, pp. 1316-1321, July 1996.

[2] S. Loyka, V. Kostina, and F. Gagnon, "Error rates of the maximumlikelihood detector for arbitrary constellations: convex/concave behavior and applications," IEEE Trans. Inf. Theory, vol. 56, no. 4, pp. 19481960, Apr. 2010.

[3] C. Goken, S. Gezici, and O. Arikan, "Optimal stochastic signaling for power-constrained binary communications systems," IEEE Trans. Wireless Commun., vol. 9, no. 12, pp. 3650-3661, Dec. 2010.

[4] — - "Optimal signaling and detector design for power-constrained binary communications systems over non-Gaussian channels," IEEE Commun. Lett., vol. 14, no. 2, pp. 100-102, Feb. 2010.

[5] M. E. Tutay, S. Gezici, and O. Arikan, "Stochastic signal design on the downlink of a multiuser communications system," in Proc. 2012 IEEE Int. Workshop on Sig. Processing Advances in Wireless Commun., pp. 75-79.

[6] A. Patel and B. Kosko, "Optimal noise benefits in Neyman-Pearson and inequality-constrained signal detection," IEEE Trans. Signal Process., vol. 57, no. 5, pp. 1655-1669, May 2009.

[7] B. Dulek and S. Gezici, "Detector randomization and stochastic signaling for minimum probability of error receivers," IEEE Trans. Commun., vol. 60, no. 4, pp. 923-928, Apr. 2012.
[8] H. Chen, P. K. Varshney, S. M. Kay, and J. H. Michels, "Theory of the stochastic resonance effect in signal detection - part II: variable detectors," IEEE Trans. Signal Process., vol. 56, no. 10, pp. 5031-5041, Oct. 2007.

[9] — - "Theory of the stochastic resonance effect in signal detectionpart I: fixed detectors," IEEE Trans. Signal Process., vol. 55, no. 7, pp. 3172-3184, July 2007.

[10] H. Chen, P. K. Varshney, and J. H. Michels, "Noise enhanced parameter estimation," IEEE Trans. Signal Process., vol. 56, no. 10, pp. 5074 5081, Oct. 2008.

[11] S. Bayram and S. Gezici, "Noise-enhanced $M$-ary hypothesis-testing in the minimax framework," in Proc. 2009 International Conference on Signal Processing and Commun. Systems, pp. 31-36.

[12] S. Bayram, S. Gezici, and H. V. Poor, "Noise enhanced hypothesistesting in the restricted Bayesian framework," IEEE Trans. Signa; Process., vol. 58, no. 8, pp. 3972-3989, Aug. 2010.

[13] M. D. McDonnell, "Is electrical noise useful?" Proc. IEEE, vol. 99, no. 2, pp. 242-246, Feb. 2011.

[14] G. Guo, M. Mandal, and Y. Jing, "A robust detector of known signal in non-Gaussian noise using threshold systems," Signal Process., vol. 92, pp. 2676-2688, 2012.

[15] E. G. Larsson, "Improving the frame-error-rate of spatial multiplexing in block fading by randomly rotating the signal constellation," IEEE Commun. Lett., vol. 8, no. 8, pp. 514-516, Aug. 2004.

[16] - "Constellation randomization (CoRa) for outage performance improvement on MIMO channels," in Proc. 2004 IEEE Global Telecommunications Conference, vol. 1, pp. 386-390.

[17] Y. Li, C. N. Georghiades, and G. Huang, "Transmit diversity over quasi-static fading channels using multiple antennas and random signal mapping," IEEE Trans. Commun., vol. 51, no. 11, pp. 1918-1926, Nov. 2003.

[18] C. Lamy and J. Boutros, "On random rotations diversity and minimum MSE decoding of lattices," IEEE Trans. Inf. Theory, vol. 46, pp. 1584 1589, July 2000.

[19] A. Hiroike, F. Adachi, and N. Nakajima, "Combined effects of phase sweeping transmitter diversity and channel coding," IEEE Trans. Veh. Technol., vol. 41, pp. 170-176, May 1992.

[20] X. Ma and G. B. Giannakis, "Space-time-multipath coding using digital phase sweeping," in Proc. 2002 IEEE Global Communications Conference, vol. 1, pp. 384-388.

[21] B. Dulek and S. Gezici, "Optimal stochastic signal design and detector randomization in the Neyman-Pearson framework," in 2012 IEEE Int. Conf. Acoustics, Speech and Signal Process.

[22] E. L. Lehmann, Testing Statistical Hypotheses, 2nd ed. Chapman \& Hall, 1986.

[23] S. M. Kay, "Noise enhanced detection as a special case of randomization," IEEE Signal Process. Lett., vol. 15, pp. 709-712, 2008.

[24] D. M. Novakovic and M. L. Dukic, "Evolution of the power control techniques for DS-CDMA toward 3G wireless communication systems," IEEE Commun. Surveys \& Tuts., vol. 3, no. 4, pp. 2-15, 2010.

[25] S. Koskie and Z. Gajic, "SIR-based power control algorithms wireless CDMA networks: an overview," Dynamics of Continuous, Discrete and Impulsive Systems B: Applications and Algorithms, no. 10-S, p. 286, 2003.

[26] M. Chiang, P. Hande, T. Lan, and C. W. Tan, "Power control in wireless cellular networks," Foundations and Trends in Networking, Now Publishers Inc., 2008.

[27] Y. Shi, R. K. Mallik, and K. B. Letaief, "Randomized power control for two-hop interference channels," IEEE Commun. Lett., vol. 14, no. 12, pp. 1128-1130, Dec. 2010.

[28] N. Tarhuni, M. Elmusrati, and A. Abouda, "Two-state discretized transmit power control for ad-hoc sensor networks," in Proc. 2011 International Congress on Ultra Modern Telecommunications and Control Systems and Workshops, pp. 1-6.

[29] T.-S. Kim and S.-L. Kim, "Random power control in wireless ad hoc networks," IEEE Commun. Lett., vol. 9, no. 12, pp. 1046-1048, Dec. 2005.

[30] M. Elmusrati, N. Tarhuni, and R. Jantti, "Performance analysis of random uniform power allocation for wireless networks in Rayleigh fading channels," Eur. Trans. Telecommun., vol. 20, pp. 457-462, 2009.

[31] _ " "Random power control for uncorrelated Rayleigh fading channels," in Proc. 2007 IEEE International Conference on Signal Processing and Communications, pp. 360-363.

[32] Y. Zhu, Y. Xu, S. Guan, and J. He, "Connectivity analysis of random power control in wireless sensor networks," in Proc. 2010 IEEE International Conference on Communication Technology, pp. 1248-1251.

[33] S. Verdu, Multiuser Detection, 1st ed. Cambridge University Press, 1998. 
[34] A. Goldsmith, Wireless Communications. Cambridge University Press, 2005.

[35] S. A. Grandhi, J. Zander, and R. Yates, "Constrained power control," Wireless Personal Commun., vol. 1, no. 4, pp. 257-270, 1995.

[36] C. W. Tan, M. Chiang, and R. Srikant, "Maximizing sum rate and minimizing MSE on multiuser downlink: optimality, fast algorithms and equivalence via max-min SINR," IEEE Trans. Signal Process., vol. 59, no. 12, pp. 6127-6143, Dec. 2011.

[37] C. Yang and J. Li, "Mixed-strategy based discrete power control approach for cognitive radios: a matrix game-theoretic framework," in Proc. 2010 International Conference on Future Computer and Communication, vol. 3, pp. V3-806-V3-810.

[38] T. Heikkinen, "A minimax game of power control in a wireless network under incomplete information," DIMACS Technical Report 99-43, Tech. Rep., 1999.

[39] H. V. Poor, An Introduction to Signal Detection and Estimation. Springer-Verlag, 1994.

[40] R. T. Rockafellar, Convex Analysis. Princeton University Press, 1968.

[41] I. Kra and S. R. Simanca, "On circulant matrices," Notices of the American Mathematical Society, vol. 59, no. 3, pp. 368-377, Mar. 2012.

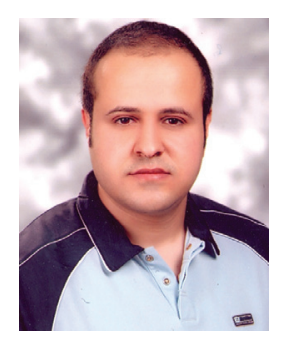

Mehmet Emin Tutay received the B.S. degree in 2008 and the M.S. degree in 2010, both from the Department of Electrical and Electronics Engineering, Bilkent University, Turkey. He is currently working towards the Ph.D. degree in the same department. His main research interests are in the fields of statistical signal processing and wireless communications.

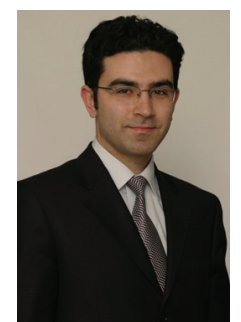

Sinan Gezici (S'03, M'06, SM'11) received the B.S. degree from Bilkent University, Turkey in 2001, and the Ph.D. degree in Electrical Engineering from Princeton University in 2006. From 2006 to 2007, he worked at Mitsubishi Electric Research Laboratories, Cambridge, MA. Since 2007, he has been with the Department of Electrical and Electronics Engineering at Bilkent University, where he is currently an Associate Professor. Dr. Gezici's research interests are in the areas of detection and estimation theory, wireless communications, and localization systems. Among his publications in these areas is the book Ultra-wideband Positioning Systems: Theoretical Limits, Ranging Algorithms, and Protocols (Cambridge University Press, 2008). Dr. Gezici is an associate editor for IEEE TRANSACTIONS ON COMMUNICATIONS, IEEE WIRELESS COMMUNICATIONS LETTERS, and the Journal of Communications and Networks.

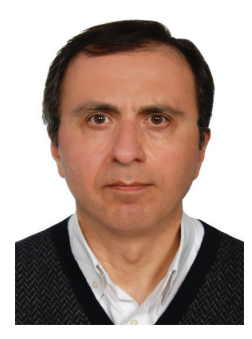

Orhan Arikan (M'91) was born in 1964 in Manisa, Turkey. In 1986, he received his B.Sc. degree in Electrical and Electronics Engineering from the Middle East Technical University, Ankara, Turkey. He received both his M.S. and Ph.D. degrees in Electrical and Computer Engineering from the University of Illinois, Urbana-Champaign, in 1988 and 1990, respectively. Following his graduate studies, he was employed as a Research Scientist at SchlumbergerDoll Research Center, Ridgefield, CT. In 1993 he joined the Electrical and Electronics Engineering Department of Bilkent University, Ankara, Turkey. Since 2011, he is serving as the department chairman. His current research interests include statistical signal processing, time-frequency analysis and remote sensing. Dr. Arikan has served as Chairman of IEEE Signal Processing Society Turkey Chapter and President of IEEE Turkey Section. 